\title{
Placeholder factors in ribosome biogenesis: please, pave
} my way

\author{
Francisco J. Espinar-Marchena, Reyes Babiano ${ }^{1}$ and Jesús de la Cruz* \\ Instituto de Biomedicina de Sevilla (IBiS), Hospital Universitario Virgen del Rocío/CSIC/Universidad de Sevilla, and Departamento de \\ Genética, Universidad de Sevilla, E-41013, Seville, Spain. \\ ${ }^{1}$ Present address: Swiss Institute for Experimental Cancer Research (ISREC), School of Life Sciences, Ecole Polytechnique Fédérale de \\ Lausanne (EPFL), CH-1015 Lausanne, Switzerland. \\ * Corresponding Author: \\ Jesús de la Cruz, Instituto de Biomedicina de Sevilla (IBiS), Campus Hospital University "Virgen del Rocío", Avda. Manuel Siurot, s/n; \\ E-41013 Sevilla, SPAIN; Phone: +34 9559231 26; Fax: +34 9559231 01; E-mail: jdlcd@us.es
}

\begin{abstract}
The synthesis of cytoplasmic eukaryotic ribosomes is an extraordinarily energy-demanding cellular activity that occurs progressively from the nucleolus to the cytoplasm. In the nucleolus, precursor rRNAs associate with a myriad of trans-acting factors and some ribosomal proteins to form preribosomal particles. These factors include snoRNPs, nucleases, ATPases, GTPases, RNA helicases, and a vast list of proteins with no predicted enzymatic activity. Their coordinate activity orchestrates in a spatiotemporal manner the modification and processing of precursor rRNAs, the rearrangement reactions required for the formation of productive RNA folding intermediates, the ordered assembly of the ribosomal proteins, and the export of pre-ribosomal particles to the cytoplasm; thus, providing speed, directionality and accuracy to the overall process of formation of translation-competent ribosomes. Here, we review a particular class of trans-acting factors known as "placeholders". Placeholder factors temporarily bind selected ribosomal sites until these have achieved a structural context that is appropriate for exchanging the placeholder with another site-specific binding factor. By this strategy, placeholders sterically prevent premature recruitment of subsequently binding factors, premature formation of structures, avoid possible folding traps, and act as molecular clocks that supervise the correct progression of pre-ribosomal particles into functional ribosomal subunits. We summarize the current understanding of those factors that delay the assembly of distinct ribosomal proteins or subsequently bind key sites in pre-ribosomal particles. We also discuss recurrent examples of RNA-protein and protein-protein mimicry between rRNAs and/or factors, which have clear functional implications for the ribosome biogenesis pathway.
\end{abstract}

doi: 10.15698/mic2017.05.572 Received originally: 13.03.2017; in revised form: 24.04.2017, Accepted 24.04.2017, Published 27.04.2017.

Keywords: ribosome assembly, ribosomal proteins, RNA mimicry, trans-acting factors, yeast.
Abbreviations:
CRAC-cross-linking and CDNA analysis,
$E M$ - electron microscopy,
GAC-GTPase-associated centre, LSU - large subunit,
MetAP - methionine aminopeptidase, NLS - nuclear localization signal, PET-polypeptide exit tunnel, pre-rRNA- precursor rRNA, PTC - peptidyl transferase centre, r-protein-ribosomal protein, rRNA - ribosomal RNA, SnoRNA - small nucleolar RNA, SSU - small subunit.

\section{INTRODUCTION}

Ribosomes are complex ribonucleoprotein organelles that are responsible for protein synthesis. In all organisms, ribosomes are composed of two ribosomal subunits ( $r$ subunits), the large one (LSU) being about twice the size of the small one (SSU) [1]. The production of ribosomes is an extraordinarily complicated cellular challenge. All organisms invest an important percentage of their resources to produce and subsequently assemble the individual constit- uents of the ribosomes, ribosomal RNAs (rRNAs) and ribosomal proteins (r-proteins), which must be represented in equimolecular amounts [2-4]. This process, known as the ribosome biogenesis pathway, is a highly coordinated process that, in addition to the rRNAs and r-proteins, involves RNA and protein trans-acting factors. Trans-acting factors transiently bind to pre-ribosomal particles in a distinctive spatiotemporal manner and have precise functions during (i) the transcription, processing and chemical modification 
of the precursor rRNAs (pre-rRNAs); (ii) the folding and rearrangements of the pre-rRNAs within the pre-ribosomal particles; (iii) the synthesis, dedicated chaperoning, nuclear import (only in eukaryotes), assembly and repositioning within pre-ribosomal particles of the different r-proteins; (iv) also in eukaryotes, the intranuclear transport, acquisition of export competence and the exit of pre-ribosomal particles to the cytoplasm, as well as the cytoplasmic maturation steps that newly synthesized ribosomes must undergo before entering translation.

In bacteria, only about a dozen of protein trans-acting factors have been reported to participate in ribosome biogenesis [4-6]. In archaea, about 50 small RNAs (i.e. modification guide small RNAs) and 40 protein trans-acting factors have been described $[5,7,8]$. In eukaryotes, however, ribosome biogenesis has clearly increased its complexity. Thus, in the yeast Saccharomyces cerevisiae, about 80 small nucleolar RNAs (snoRNAs) and more than 250-300 protein trans-acting factors are currently known to participate in this process $[9,10]$. In humans, about 300 snoRNAs and more than 600 protein factors have been shown so far to be required for the biogenesis of ribosomes [11, 12].

Ribosome synthesis is clearly a directional process [13]. Thus, most reactions occur irreversibly during the maturation of pre-ribosomal particles, among them, the pre-rRNA processing steps, the pre-rRNA folding, the snoRNAdependent modifications, and the stepwise exchange of some protein trans-acting factors (for a general scheme of the ribosome biogenesis process, see Figure S1). Eukaryotes have evolved a nucleus, therefore, pre-ribosomal particles must travel across the nucle(ol)us and, after the acquisition of export competence, exit to the cytoplasm. Different factors are part of a quality control machinery that allows export-competent pre-ribosomal particles to associate with transport factors, which mediate their interactions with the nuclear pore complexes and their transport to the cytoplasm [14]. Moreover, although it has been suggested that some translation might occur in the nucleus [15], it seems clear that, at least, nascent nuclear pre-ribosomal particles are incompetent for translation until the release of the last protein trans-acting factors in the cytoplasm and the assembly of the last r-proteins [16-18]. These events, the displacement of the last factors and the assembly of the last $r$-proteins in the cytoplasm, are apparently prerequisites to confer translational competence to $r$-subunits (i.e. [19-21]).

Several protein trans-acting factors have been reported that act as placeholders. The word "placeholder" has different meanings, among others it refers to someone who occupies a professional position on behalf of someone else. In Molecular Biology, a placeholder corresponds to a factor that temporarily binds a target until its replacement by a second factor, which binds to the same target normally with a higher affinity. Placeholder factors have been described to participate in different cellular processes, including chromatin remodelling and transcription (e.g. [22-24]). In this review, we outline the current knowledge about placeholder factors involved in the biogenesis of ribosomes, focusing primarily on those from the yeast $S$. cerevisiae, in which this process has been most extensively studied. These factors include trans-acting factors, such as Mrt4 or RIp24, which are paralogous placeholders of distinct $r$ proteins, or others trans-acting factors, such as Arx1, Nog1, Nog2/Nug2 or Tsr1, whose replacement pairs are not $r$ proteins. We also examine the role of distinct adaptors and chaperones, such as Rrb1, Sqt1 or Yar1, which recognize domains on their r-protein partners that are normally involved in binding to rRNAs. This latter phenomenon also resembles that known as RNA mimicry, by which some factors, such as Fap7 or Syo1, interact with a specific $r$ protein through the establishment of a protein interface that imitates part of the rRNA-binding surface of these $r$ proteins. We emphasise how all these factors, whose functions during ribosome biogenesis expand in many cases beyond their placeholding activity, and strategies render properly assembled $r$-subunits competent for translation.

\section{THE CLASSICAL VIEW: PARALOGUES OF RIBOSOMAL PROTEINS}

In yeast, most r-proteins genes are duplicated and encode identical or nearly identical paralogous r-proteins, which, apparently in numerous cases, are functionally redundant [25]. Strikingly, few r-proteins have additional paralogues that share extensive identity and similarity to them (e.g. [26-28]). These paralogues, also known as ribosomal-like proteins, are not natural components of mature ribosomes and are unable to functionally replace their r-protein counterparts, even when overexpressed (e.g. [26, 29]), but interestingly, all of them have a role during ribosome biogenesis [28, 30-32]. This fact prompted S. J. Baserga to propose that each ribosomal-like protein could act as a placeholder for its paralogous r-protein on the pre-rRNA. A placeholder factor acts by preventing the premature assembly of its r-protein counterpart on its rRNA binding site, which, both proteins, considering their extensive homology, may share [31].

Ribosomal-like proteins include Imp3, Mrt4, Rlp7 and RIp24, which display considerable sequence homology to $r$ proteins S9 (uS4 according to the recently proposed $r$ protein nomenclature [33]), PO (uL10), L7 (uL30) and L24 (eL24), respectively. In addition, part of the Nob1 endonuclease exhibits significant homology to S26 (eS26) [17] (Figure S2). Moreover, several trans-acting factors have gained ancient RNA-binding motifs in their structures that resemble those present in distinct $r$-proteins; for instance, Rrp5 contains 12 tandem S1 (bS1) RNA-binding motifs in its $\mathrm{N}$-terminal domain [34]; Snu13 is member of a family of Kturn binding proteins that also includes human $r$-proteins L7A (eL8) and S12 (eS12), and yeast r-protein L30 (eL30) [35]. In this section, we discuss the functional relationship of Mrt4, Rlp24, Rlp7 and Imp3 with their paralogous $r$ proteins. The implications of the homology between Nob1, Rrp5 or Snu13 and their respective r-protein counterparts will not be discussed. In these three cases, it remains to be determined whether the trans-acting factors could have a placeholder activity. 
A

Pre-60S (Nog2-TAP)

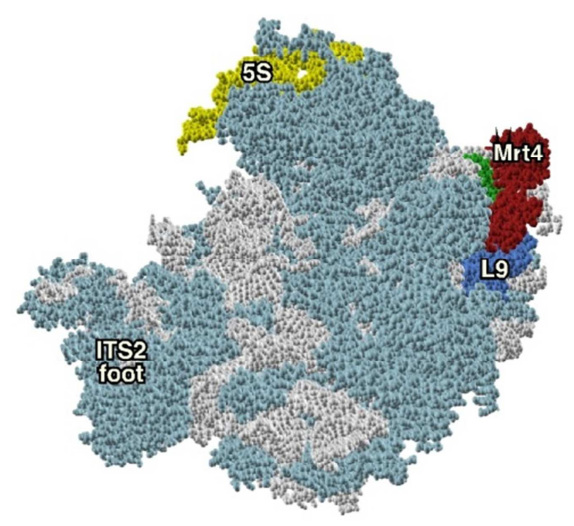

B

Pre-60S (Nmd3-TAP)

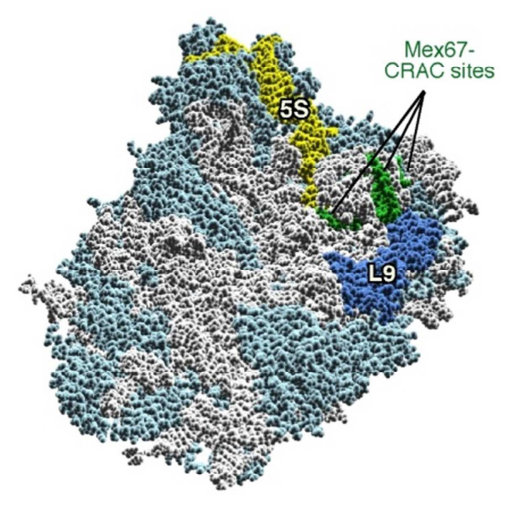

C

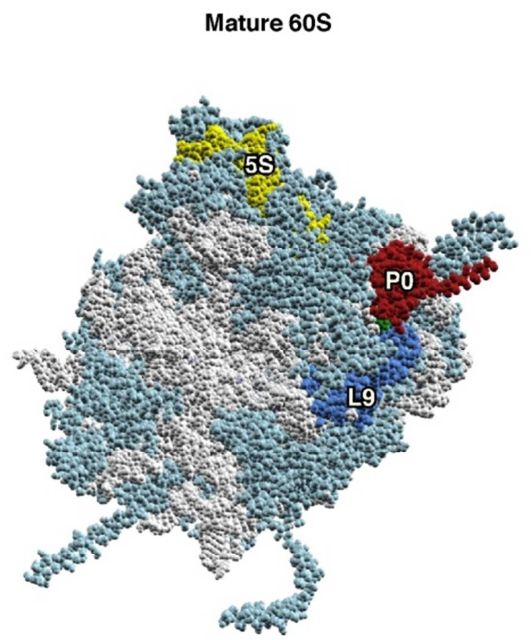

Figure 1: Mrt4 and Mex67 act as placeholder factors for the P0 r-protein. (A) Position of Mrt4 (red) in the early pre-60S r-particles purified with Nog2-TAP (PDB ID: 3JCT; [40]). (B) Mex67-binding sites at the P0 neighbourhood (green), identified by CRAC [42], have been highlighted in the late/cytoplasmic Nmd3-TAP pre-60S r-particle (PDB ID: 5H4P; [43]. (C) Position of P0 (red) in the mature 60S r-subunit (PDB ID: 3U5I, $3 \mathrm{U} 5 \mathrm{H}$; [44]). Particles are viewed from the subunit interface slightly turned to the left. For orientation, the positions of the 5S rRNA (yellow), the L9 r-protein (royal blue) and the above CRAC sites of Mex67 (green) have been highlighted in the three structures. The ITS2 foot has also labelled in A. Note that some of the CRAC sites of Mex67 overlap with Mrt4 and PO in A and C, respectively. The rest of rRNAs are coloured in pale blue and the rest of r-proteins and/or factors in light cornflower blue. Images were generated using the UCSF Chimera program (www.cgl.ucsf.edu/chimera).

\section{Mrt4 and Mex67 versus P0}

From all these examples, perhaps the best-studied, homology-sharing pair of proteins consists of Mrt4 and P0. Mrt4 is homologous to the N-terminal domain of PO (Figure S2), which corresponds to the rRNA binding domain of the $r$ protein [36]. PO has an additional C-terminal extension that is exposed to the solvent and interacts with the acidic P1 and $\mathrm{P} 2 \mathrm{r}$-proteins and translation elongation factors [37]. A few years ago, we could show that yeast PO and Mrt4 are unable to bind simultaneously to $r$-particles by analysing the presence of either protein in complexes purified using functional TAP-tagged Mrt4 or PO as affinity baits, respectively [29]. This observation, together with the fact that a Mrt4-P0 chimera protein, containing as $\mathrm{N}$-terminal domain the Mrt4 ORF, is able to partially complement the otherwise lethal absence of PO [29], and that a truncated PO rprotein lacking its C-terminal domain functionally resembles Mrt4 [38], strongly suggest that P0 and Mrt4 compete for the same rRNA site in r-particles, thus, successively occupying this site during LSU maturation [29]. A similar scenario has been reported for human Mrt4 and PO [39]. In full agreement with this hypothesis, the cryo-electron microscopy (cryo-EM) reconstruction of two distinct yeast pre-60S r-particles, which carry Mrt4 but lack P0, has revealed that indeed in these particles Mrt4 unequivocally localizes to a position equivalent to the one of $\mathrm{PO}$ in the $\mathrm{P}$ stalk of the mature LSU (Figures $1 A$ and $1 C$ ) $[40,41]$. Strikingly, theoretical estimation of the free RNA binding energy of both proteins suggests that PO might bind to its rRNA site a little tighter than Mrt4 [29], a fact whose biological significance will be further discussed.
The dynamics of the sequential exchange reaction of Mrt4 with P0 have been studied in vivo. Different evidence indicates that Mrt4 is a nucleo-cytoplasmic shuttling assembly factor, which associates with early to intermediate pre-60S r-particles and predominantly dissociates from late, cytoplasmic pre-60S r-particles [20, 45]. The replacement of Mrt4 by P0, thus, takes place mostly in the cytoplasm, although it could also occur in the nucleus [38]. This replacement is a prerequisite to recycle Mrt4 back to the nucle(ol)us [20]. How exactly this reaction takes place mechanistically is still unknown. Moreover, the exchange does apparently not occur directly, but instead, requires the participation of Yvh1, which is another nucleocytoplasmic shuttling assembly factor, non-homologous to either Mrt4 or P0 that co-enriches with late/cytoplasmic pre-60S r-particles [42, 45, 46]. Interestingly, Yvh1containing r-particles do neither contain Mrt4 nor P0 and equivalent results are obtained in reciprocal experiments $[42,45,46]$. However, whether Yvh1 competes with the rRNA-binding site of Mrt4 and PO has not been addressed until very recently [42]. It has been shown that the stable association of Yvh1 with pre-60S r-particles depends on the r-protein L12 (uL11), which is the closest neighbour of P0 at the base of the P-stalk $[44,47]$. However, still in the absence of L12, there is apparently no difference in the efficiency of Mrt4 re-importation to the nucleus ([48], and our unpublished results). In conclusion, despite the fact that yeast Mrt4 and Yvh1 are non-essential proteins under standard laboratory conditions, both factors may play important roles controlling the position and timing of the assembly of $\mathrm{PO}$, simultaneously providing a surveillance 
point to ensure that only mature LSUs can engage in translation (see below; further discussed in [14, 17, 20]).

Interestingly, the Hurt laboratory has recently described that the nuclear-export factor Mex67 is another placeholder of the P0 r-protein [42], even though, Mex67 barely displays sequence homology with either Mrt4 or P0 (Figure S2). This group has identified that the heterodimeric Mex67•Mtr2 complex, which is involved in the export of late pre-60S r-particles $[49,50]$, binds in vitro at two distant positions on Yvh1-purified pre-60S r-particles; the first one overlaps with the rRNA-binding site within the $5.8 \mathrm{~S}$ rRNA of the RNA helicase Mtr4/Dob1, which is a cofactor of the exosome complex responsible of the 3 ' end maturation of $7 \mathrm{~S}$ pre-rRNAs to $5.8 \mathrm{~S}$ rRNAs ([51]; for a review, see [52]); strikingly, the second position overlaps with the binding site of Mrt4 and PO in pre-60S r-particles and the mature LSU, respectively (Figure 1B) [42]. Remarkably, it could be shown that the Mex67•Mtr2 complex can hardly bind late pre-60S r-particles containing Mrt4 in vitro; this result is in agreement with a competition between Mex67•Mtr2 and Mrt4 for the same binding site if assuming only a minor contribution of the Mex67•Mtr2 rRNAbinding site at the 5.8S rRNA in these particles [42]. Moreover, the structural characterization of Yvh1-containing pre-60S r-particles by cryo-EM reveals that Yvh1 binds adjacent to L12, a position that is close to but apparently not mutually exclusive to those of Mex67•Mtr2, Mrt4 or P0 [42].

A

\section{Pre-60S (Nog2-TAP)}

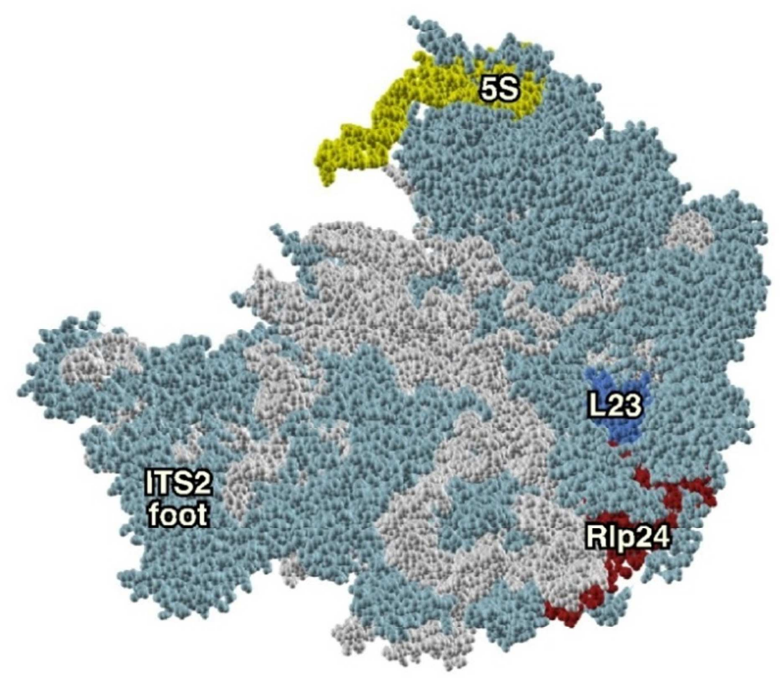

Taken together, the following model for the timing of PO assembly has been proposed: (i) Mrt4 binds first at the incipient P-stalk site of nuclear pre-60S r-particles. (ii) Later, but still in the nucleus, the heterodimer Mex67-Mtr2 bound to Yvh1 replaces Mrt4 in the pre-60S r-particles; then, Mex67•Mtr2 acts as one of the RanGTP-independent factors involved in the export of pre-60S r-particles to the cytoplasm; (iii) once in the cytoplasm, the assembly of PO occurs concomitantly to the release of Mex67•Mtr2 and Yvh1 from the base of the P-stalk in the cytoplasmic pre605 r-particles. How can we reconcile this model with apparently contradictory findings indicating that the release of Mrt4 takes place mostly in the cytoplasm? The answer is not obvious, but the scenario clearly suggests the existence of alternative pathways to perform the same reaction. Moreover, as nuclear export of pre-60S r-particles is mediated by several redundant but cooperative systems (reviewed in [14]) and Yvh1 is a non-essential factor [45, 46], it is conceivable that Mrt4 may exchange in the cytoplasm, not only independently of Mex67•Mtr2, but also of Yvh1.

\section{Rlp24 versus L24}

Another well-studied paralogous pair comprised of a ribosomal-like protein and an r-protein is represented by the conserved eukaryotic trans-acting factor RIp24 and the LSU r-protein L24. Rlp24 and L24 share the N-terminal domain, which for L24 corresponds to the region that binds to mature LSUs (Figure S2). Yeast Rlp24 belongs to the category

\section{B}

\section{Mature 60S}

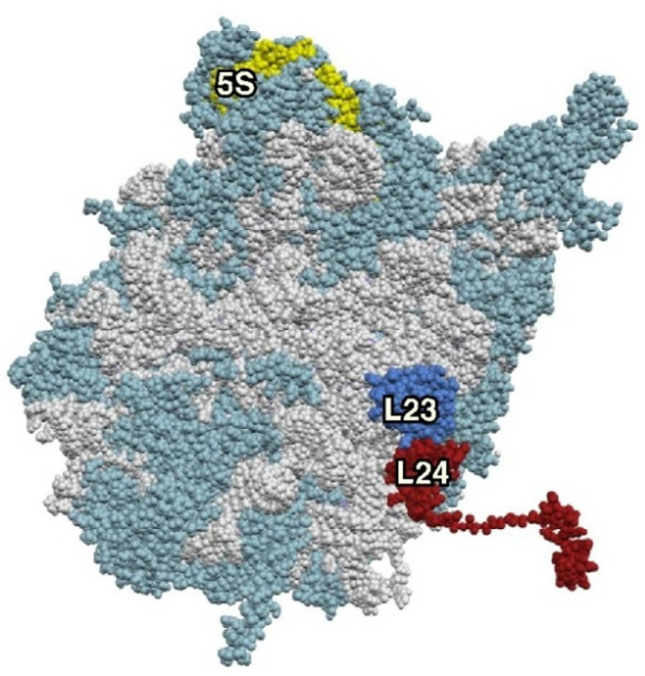

Figure 2: Rlp24 functions as a placeholder factor for the L24 r-protein. (A) Position of Rlp24 (red) in the early pre-60S r-particles purified with Nog2-TAP (PDB ID: 3JCT; [40]). (B) Position of L24 r-protein (red) in the mature 60S r-subunit (PDB ID: 3U5I, 3U5H; [44]). Particles are viewed from the subunit interface. For orientation, the positions of the 5 S rRNA (yellow) and the L23 r-protein (royal blue) have been highlighted. The ITS2 foot has also labelled in A. Note that the last ca. 50 amino acids from the C-terminal part of Rlp24 and ca. 20 amino acids from the C-terminal end of L24 could not be modelled in the respective structures. The rest of rRNAs are coloured in pale blue and the rest of $r$-proteins and/or factors in light cornflower blue. 
of trans-acting factors known as B factors, which are required for the proper maturation of 27SB pre-rRNAs within intermediate pre-60S r-particles [53]. L24 is a non-essential LSU r-protein, whose role in LSU biogenesis has so far not been properly characterized [28]. In any case, there is enough evidence to conclude that Rlp24 functions as a bona fide placeholder for L24. First, L24 is not present in pre-60S r-complexes purified using TAP-tagged RIp24 as a bait [28]. Second, both proteins seem to recognize the same binding site on $r$-particles, as shown by cryo-EM studies of distinct nuclear pre-60S r-particles, which reveal that the density found at the location of L24 in these particles clearly corresponds to the N-terminal part of RIp24 (Figure 2) $[40,41]$. As a corollary of this, release of Rlp24 from pre$60 \mathrm{~S} \mathrm{r}$-particles is a pre-requisite for assembly of L24.

The replacement of RIp24 with L24 occurs in the cytoplasm following different steps: (i) Rlp24, most likely assisted by the WD-40 repeat protein Mak11, associates in the nucleolus with very early pre-60S r-particles $[28,54]$. This reaction appears to be coupled to the recruitment of the GTPase Nog1 to pre-60S r-particles, which directly and specifically interacts with Rlp24 [28, 40]. (ii) Upon arrival in the cytoplasm, the AAA-ATPase Drg1, which forms hexamers in the presence of ATP, binds to the exported pre$60 \mathrm{~S}$ r-particles and allows the specific dissociation of Rlp24. This dissociation step is a prerequisite for the subsequent cytoplasmic maturation steps of these pre-60S r-particles, including the release of other shuttling factors such as Nog1 and Bud20 and the recruitment of later-acting cytoplasmic factors such as Rei1 [55-58]. A monomer of Drg1 is composed of an N-terminal domain followed by two consecutive AAA-ATPase domains: D1 and D2 (reviewed in [59]). In vitro, Drg1 binds specifically and directly to the Cterminal domain of Rlp24, which is not conserved in L24 [60]; consistently, expression of a truncated version of Rlp24 lacking the last 53 amino acids (RIp24 $\Delta C$ ) prevents the recruitment of Drg1 to pre-60S r-particles in vivo, and, as a consequence, RIp $24 \Delta C$ is not properly released from cytoplasmic pre-60S r-particles and final maturation of nascent LSUs cannot be completed [55]. In vitro, the Cterminal domain of Rlp24 also stimulates ATP hydrolysis in both AAA domains of Drg1 [60]; while ATP hydrolysis in the D2 domain triggers the dissociation of Rlp24 from pre-60S $r$-particles, ATP hydrolysis in the D1 domain is required for the subsequent release of Drg1 from RIp24 and likely the dissociation of the Drg1 hexamer into monomers [60, 61]. Interestingly, it has been shown that Drg1 also directly binds the FG-repeat nucleoporin Nup116, and more importantly, that this interaction optimizes the release of RIp24 from pre-60S r-particles, suggesting some coupling between the export of pre-60S r-particles and the initiation of their cytoplasmic maturation [60]. (iii) Finally, RIp24 is recycled back to the nucle(ol)us and L24 stably assembles into pre-60S r-particles. However, in clear contrast to P0, which is required for the efficient release of its placeholder Mrt4 from pre-60S r-particles [20], likely indirectly by its role in dissociating Yvh1 and the Mex67-Mtr2 complex from those particles [55], it appears that L24 does not contribute to the release of Rlp24 from pre-60S r-particles.
Thus, the complete absence of $L 24$, by the double deletion of the RPL24A and RPL24B genes, does not lead to a failure in either the release or the subsequent nucleolar recycling of RIp24 (cited as unpublished results in [28]).

The cytoplasmic assembly of L24 appears to be coupled to the recruitment of the non-essential factor Rei1 to pre605 r-particles [62]. Rei1 is highly homologous to Reh1, and, it has been shown that both factors have a partially redundant function during maturation of nascent LSUs $[62,63]$. Rei1 directly interacts with the J-domain protein Jjj1, which recruits and activates the Hsp70-type ATPase Ssa1-Ssa2 [64-66]. Different authors have shown that one of the primary roles of Rei1 is the release and nuclear recycling of the heterodimeric Arx1•Alb1 complex from cytoplasmic pre-60S r-particles, although it is not known how this reaction mechanistically occurs $[64,66,67]$. Moreover, this activity has indeed been questioned and attributed to either Jjj1 and Ssa [68] or Reh1 [43], which both have been suggested to release simultaneously Rei1 and Arx1. The recent cryo-EM characterization of r-particles containing Rei1, Arx1 and Jjj1 or with Rei1, Arx1 and Alb1 at nearatomic resolution $[68,69]$ will allow the development of models that clearly will help to solve this question. These and others models derived from cryo-EM reconstruction analyses of selected pre-60S r-particles (e.g. see [70]), unambiguously show that Arx1 binds near the solventexposed side of the polypeptide exit tunnel (PET), suggesting that it could function as a placeholder for different nascent chain-associated factors, including methionine aminopeptidases (MetAPs) (discussed later). Interestingly, these analyses also reveal the global structure of Rei1 on $r$ particles, notably showing that its C-terminal segment penetrates into the PET and extends almost up to the peptidyl transferase centre (PTC) [68]. Strikingly, this segment is structurally homologous to the C-terminal extension of the GTPase Nog1 [40] and to the one of Reh1 [43], which could also similarly insert into the PET; thus, the binding of these three factors to pre-60S r-particles is mutually exclusive and, as it will be discussed later, confers directionality to the cytoplasmic LSU maturation. These findings therefore indicate that Nog1 is a placeholder factor for Rei1, and in turn, Rei1 a placeholder factor for Reh1. Alternatively, Rei1 and Reh1 may have redundant functions and Nog1 could function as placeholder for either factor (see below).

\section{THE EXCEPTIONS}

Not all ribosomal-like proteins act as placeholders of their respective paralogous r-proteins. Indeed, different studies on the trans-acting factors RIp7 and Imp3 clearly contradict the intuitive hypothesis that these factors could compete with their counterpart r-proteins L7 and S9, respectively, for the same binding sites on the pre- or mature rRNAs.

\section{Rlp7 versus $L 7$}

Our group, in collaboration with that of M. Fromont-Racine and A. Jacquier, reported a few years ago that RIp7 and L7 could coexist in the same pre-60S r-particles; consistently, cross-linking and CDNA analysis (CRAC) experiments dem- 
A

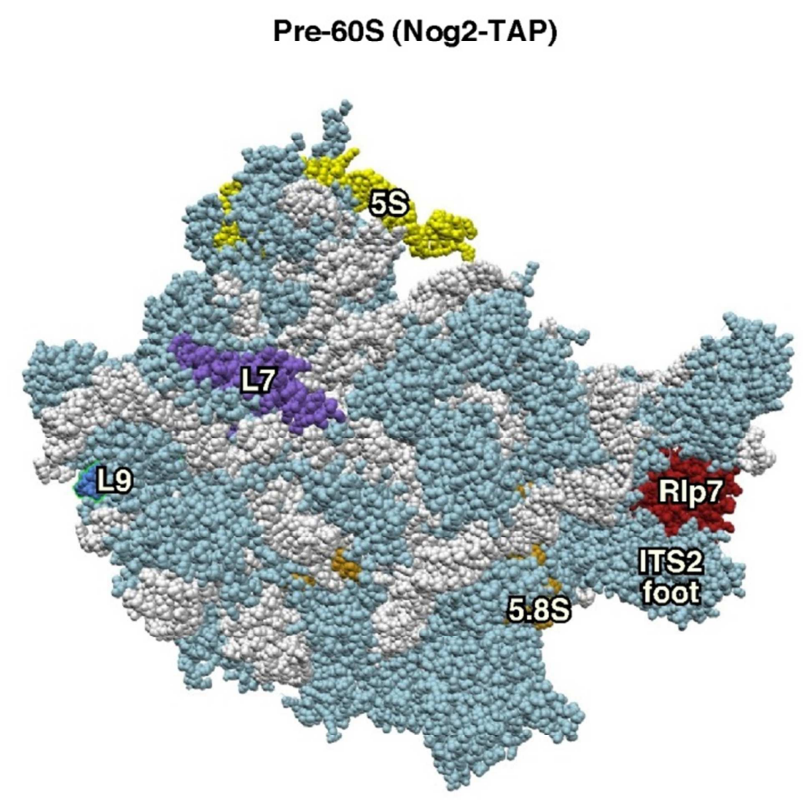

B

\section{Mature 60S}

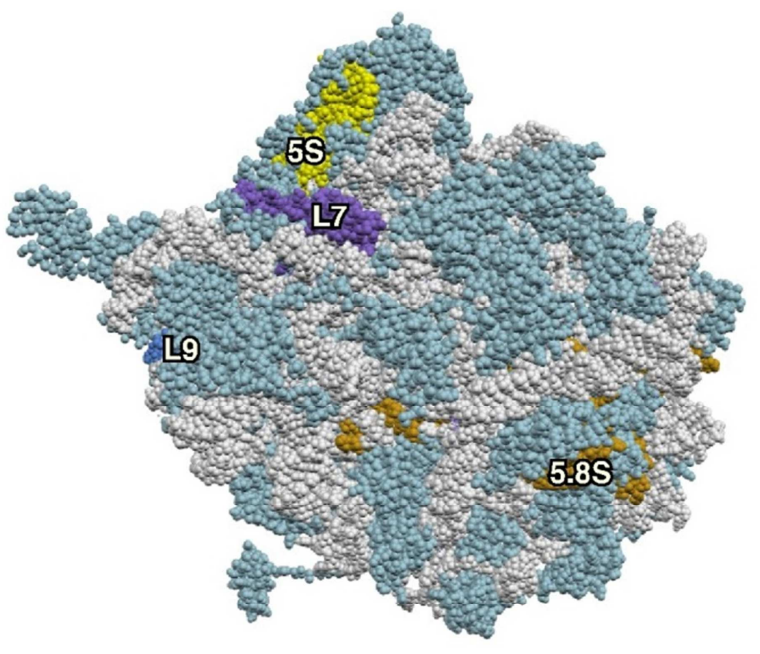

Figure 3: Rlp7 is not the placeholder factor for the assembly of L7 r-protein. (A) Position of Rlp7 (red) and L7 (purple) in the early pre-60S rparticles purified with Nog2-TAP (PDB ID: 3JCT; [40]). (B) Position of L7 r-protein (purple) in the mature 60S r-subunit (PDB ID: 3U5I, 3U5H; [44]). Particles are viewed from the solvent side. For orientation, the positions of the 5S rRNA (yellow), 5.8S rRNA (gold) and the L9 r-protein (royal blue) have been highlighted. The ITS2 foot has also labelled in A. Note that L7 is found at its final assembly position within Nog2-TAP pre-60S $r-$ particles. The first ca. 20 amino acids of both proteins could not be modelled in either structure. The rest of rRNAs are coloured in pale blue and the rest of $r$-proteins and/or factors in light cornflower blue.

onstrated that the Rlp7 and L7 binding sites are actually distinct in pre- and/or mature rRNAs and distant enough from each other that they do not result in steric binding interference [71]. Similar findings were independently obtained by the laboratory of J. L. Woolford, Jr. [72]. Rlp7, which is 78 amino acids longer than $\mathrm{L} 7$, shares a considerable overall sequence and structure homology with L7, except in its N-terminal region, its internal loop and few other discrete regions (Figure S2). Rlp7 belongs to the group of proteins known as A3 assembly factors [10], which hierarchically and interdependently associate with early pre-60S r-particles and are globally required for optimal $5^{\prime}$ to $3^{\prime}$ exonucleolytic trimming of the 27SA3 pre-rRNA to the 27SBS pre-rRNA, a processing step that generates the $5^{\prime}$ end of mature 5.8SS rRNA [10, 31, 52, 73, 74]. However, the rRNA-binding site of Rlp7, as those of the other A3 assembly factors, maps to positions in the ITS2 spacer instead of positions close to the 5 ' end of 27SA3 pre-rRNA in the ITS1 spacer $[71,72,75]$. It has been suggested that the A3 assembly factors may play structural roles in chaperoning ITS2 within pre-60S r-particles, thus protecting 275 prerRNAs from rapid turnover and facilitating their correct processing (further discussed in [10, 73, 75], see also [51]). Cryo-EM analyses have confirmed the rRNA-binding sites of Rlp7 and several other A3 factors. These sites cluster around ITS2, providing an explanation to the interdependent association of A3 factors with pre-60S r-particles [40, 41]. However, in the crystal structure of mature LSUs, the globular domain of $L 7$ binds domain II of $25 S$ rRNA, as well as $5 \mathrm{~S}$ rRNA, while its $\mathrm{N}$-terminal extension interacts with the expansion segment ES7 of 25S rRNA (Figures 3A and $3 B)[44,47]$. In agreement with our data [71], the L7 interactions already exist in pre-60S $r$-particles, as also revealed by cryo-EM analyses of selected pre-60S particles (Figures $3 A$ and $3 B)[40,41]$. Curiously, it has been shown that $L 7$ is also required for $27 \mathrm{SA} 3$ pre-rRNA processing [76-78]. It seems that the assembly of $L 7$, as that of any of its neighbouring r-proteins in rRNA domain II, such as L4 (uL4), L6 (eL6), L14 (eL14), L16 (uL13), L18 (eL18), L20 (eL20), L32 (eL32) and L33 (eL33), allows the stabilisation of rRNA structures within pre-60S $r$-particles, which is a prerequisite for the stable association and function of A3 assembly factors ([77, 79], further discussed in [80]).

Likely, Rlp7 has evolved from the highly conserved L7 rprotein to use a similar recognition motif to bind and function at a different location during LSU maturation. However, it is unclear how proteins showing apparently very similar architectures could be specifically targeted to different places in pre-ribosomal complexes, instead of competing for the same RNA substrates. As a possibility, we can imagine a scenario in which distinct co-factors specifically recruit the trans-acting factor or the corresponding paralogous $r$-protein to the pre-rRNAs at their different RNA binding sites and help their stable association or assembly. 
A

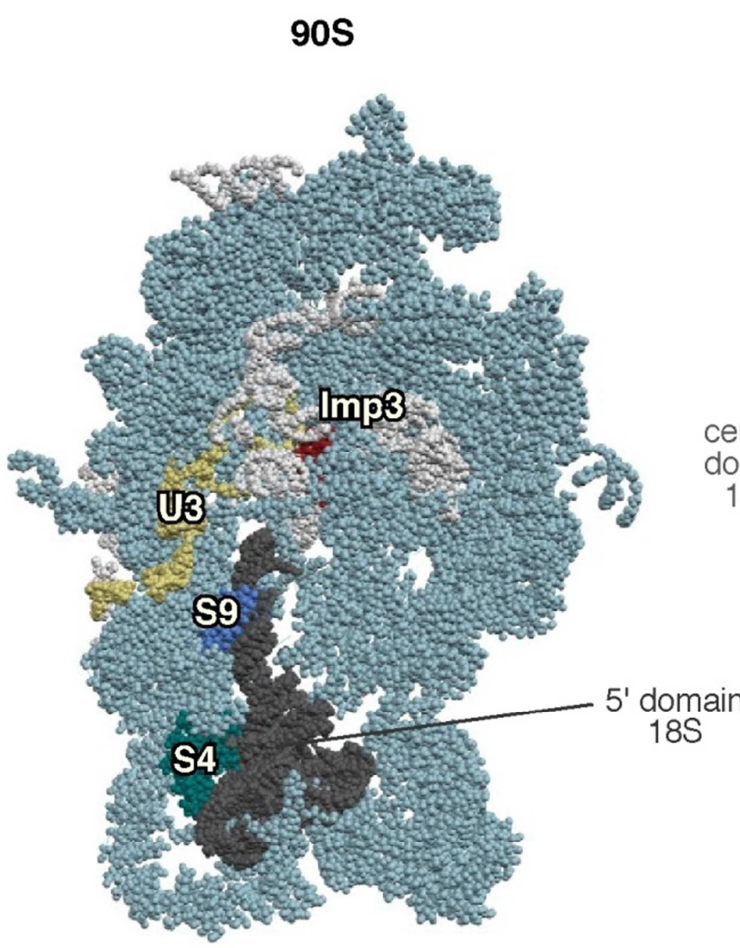

B

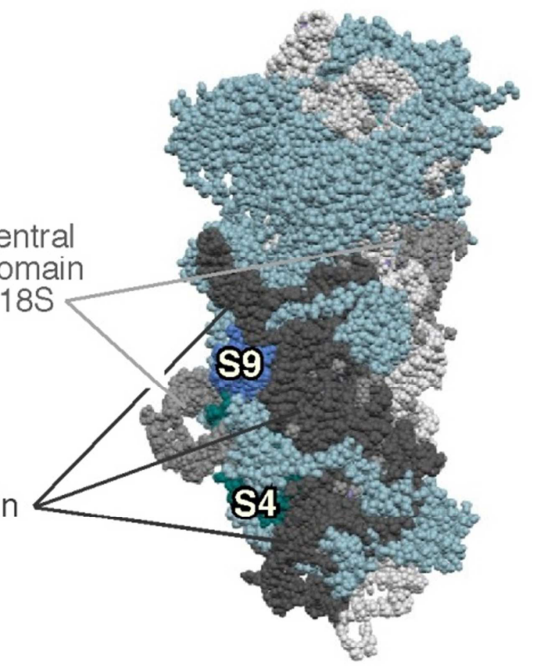

Figure 4: $I \mathrm{mp3}$ is not the placeholder factor for the assembly of S9 r-protein. (A) Position of Imp3 (red) and S9 (medium blue) in the 90S preribosomal particle, also known as the SSU processome (PDB ID: 5TZS; [93]). (B) Position of S9 rprotein (royal blue) in the mature $40 \mathrm{~S}$ r-subunit (PDB ID: 3U5B, 3U5C; [46]). Mature $40 \mathrm{~S} r$-subunit is seen from the A-site view, and the $90 \mathrm{~S}$ preribosomal particles has been consequently oriented from a similar position regarding the nascent $40 \mathrm{~S} r$-subunit. The positions of the U3 snoRNP (yellow) and that of the S4 rprotein (cyan) have been highlighted. The 5 ' domain of 18S rRNA has been coloured in dark grey, the central domain in medium grey, and the rest of $18 \mathrm{~S}$ rRNAs in pale blue; r-proteins and/or factors have been coloured in light cornflower blue.

\section{Imp3 versus S9}

Imp3 is a paralogue of the SSU r-protein S9. The similarity of these two proteins extends all over their complete sequences (Figure S2), including their putative, conserved RNA-binding domains [32]. The functional role of Imp3 has been investigated in vitro and in vivo; Imp3 is an essential trans-acting factor required for SSU biogenesis, more specifically for the cleavage at the early sites $A 0, A 1$ and $A 2$ in the $35 \mathrm{~S}$ pre-rRNA within $90 \mathrm{~S}$ pre-ribosomal particles [32]. It has also been shown that Imp3, together with the factors Mpp10 and Imp4, forms a stable sub-complex [32, 81], which co-transcriptionally associates with the 5 '-ETS region of $35 \mathrm{~S}$ pre-rRNA [82-84]. In vitro experiments have suggested that the association of this sub-complex with $90 \mathrm{~S}$ pre-ribosomal particles is required to mediate and stabilize specific base-pair interactions of residues in 5' ETS with the hinge region on the $5^{\prime}$ end of the U3 snoRNA $[85,86]$; these base pairings are indeed critical for in vivo ribosome maturation (reviewed in [87]). In turn, S9 is an essential rprotein, which is also required for early pre-rRNA processing at the A0-A2 sites [88]. In yeast and humans, S9 is a primary binding $r$-protein that assembles likely cotranscriptionally to the body of the SSU [89, 90], more specifically at positions corresponding to helices H3, H12, H17, and expansion segment ES6S of the 18S rRNA, while also interacting with other SSU r-proteins such as S2 (uS5), S4 (eS4), S24 (eS24) and S30 (eS30) (Figure 4) [44, 91]. The cryo-EM reconstruction of $90 \mathrm{~S}$ pre-ribosomal particles from both $S$. cerevisiae and Chaetomium thermophilum, an ascomycete related to $S$. cerevisiae [92], has recently been obtained [93-95]. These reconstructions have allowed the identification of the position of many trans-acting factors from these particles, among them, Imp3 and about a dozen $r$-proteins from the SSU. Some of these r-proteins have consistently been identified as stable components of purified 905 pre-ribosomal particles [96-98]. In the reconstructions, Imp3 binds to Mpp10 and Imp4, and this latter approaches the 5' part of U3 snoRNP. In agreement with its early assembly, S9 was found among those SSU r-proteins identified in the $90 \mathrm{~S}$ r-particles. Importantly, S9 appears to be bound to its final rRNA-binding site within the nascent $18 \mathrm{~S}$ rRNA, adopting a mature-like conformation in the particles (Figure 4) [93, 94]. In conclusion, all these data indicate that Imp3, despite its similarity to $\mathrm{S9}$, is not its placeholder factor in $90 \mathrm{~S}$ pre-ribosomal particles.

\section{NEW PLACEHOLDER FACTORS: THE MOST RECENT DATA}

In the last ten years, the use of CRAC and cryo-EM methodologies has permitted gaining information concerning the rRNA-binding sites and the location of a considerable number of trans-acting factors, especially from yeast, within pre-ribosomal particles. These achievements, together with the information available on the structure and location of all r-proteins revealed by crystal structures of ribosomes or r-subunits of different eukaryotes, the compositional analysis of pre-ribosomal particles and, importantly, the extended body of genetic and biochemical data on the role of trans-acting factors and r-proteins, are providing clues into the mechanistic details of the ribosome assembly process for the first time at high resolution. Particularly relevant to the scope of this review has been the discovery 
of many other examples of placeholder factors that mask particularly important ribosomal sites until a specific $r$ subunit maturation event has been accomplished. In this section, we enumerate several examples of placeholder factors that we consider to have a clear biological relevance, focusing on those that block the recruitment of essential translation factors or r-proteins to cytoplasmic preribosomal intermediates, thus, ensuring that only mature $r$ subunits engage in protein synthesis.

\section{Tsr1 blocks binding of both the GTPase elF5B and the ATPase Rio1 to late pre-40S ribosomal particles}

Tsr1 is an essential conserved trans-acting factor required for efficient nucleo-cytoplasmic transport of pre-40S $r$ particles and processing of $20 \mathrm{~S}$ pre-rRNA to mature $18 \mathrm{~S}$ rRNA $[99,100]$. Tsr1 has been shown to be recruited to early pre-40S $r$-particles in the nucleolus and accompany them together with a few other trans-acting factors (Dim1, Enp1, Ltv1, Pno1/Dim2, Nob1, and Rio2) to the cytoplasm. There, Tsr1 rapidly dissociates and is recycled back to the nucleolus $[100,101]$. Interestingly, Tsr1 is structurally related to translational GTPases such as EF-Tu or elF5B/Fun12 in their GTP-bound form. However, Tsr1 neither is a GTP-binding protein nor has GTPase activity [102]. As expected from this structural similarity, cryo-EM and CRAC analyses confirmed that Tsr1 binds, albeit differently than standard translational GTPases [102], the GTPase centre site on pre-40S r-particles [21, 102, 103]. In any case, the binding of Tsr1 to pre-40S particles is mutually exclusive with, at least, that of the GTPase elF5B and the ATPases Rli1 and Rio1, which are all required for proper maturation of pre-40S r-particles to SSUs [21, 102, 104]. Moreover, the position of Tsr1 on pre-40S r-particles potentially impedes joining of these particles to LSUs and occludes part of the mRNA channel $[21,102]$.

In conclusion, the presence of Tsr1 on pre-40S $r$ particles is incompatible not only with maturation of SSU but also with translation. Thus, Tsr1 is a good example of a placeholder factor for a set of distinct factors that times key steps during SSU formation and function. How and when the dissociation of Tsr1 from pre-40S r-particles is triggered is still unknown.

It is worth mentioning that Tsr1 shares substantial sequence identity with another GTPase, Bms1 [99]. As Tsr1, Bms1 is an essential trans-acting factor involved in SSU biogenesis, but in contrast to Tsr1, Bms1 is required for pre-rRNA processing at the early sites $A 0, A 1$ and $A 2$ [99, 105]. Bms1 is a stable component of $90 \mathrm{~S}$ pre-ribosomal particles [96] that likely binds co-transcriptionally to the nascent pre-rRNA $[83,84,106]$ and apparently efficiently dissociates following the formation of early nuclear pre$40 \mathrm{~S}$ r-particles [100]. As mentioned above, Tsr1 seems to be recruited to these type of pre-40S r-particles. Interestingly, Bms1 has been unambiguously modeled into the cryo-EM structure of $90 \mathrm{~S}$ pre-ribosomal particles [93-95]. In one of these articles, the authors have claimed that the binding site of Bms1 overlaps significantly with that of Tsr1 and suggested that Bms1 could likely work as placeholder for Tsr1 during the transition of $90 \mathrm{~S}$ to pre-40S ribosomal particles [95]. However, whether or not release of Bms1 is linked to recruitment of Tsr1 is still unexplored.

\section{Molecular events involving $\mathrm{Nmd3}$}

$\mathrm{Nmd3}$ is an essential conserved factor that connects pre$60 \mathrm{~S}$ r-particles to the $\mathrm{Crm} 1 / \mathrm{Xpo} 1$ exportin by its nuclear export sequence (NES), thereby enabling the RanGTPdependent export of late pre-60S r-particles from the nucleus $[107,108]$. Nmd3 exits to the cytoplasm associated with pre-60S r-particles where it is released and then recycled back [107]. It has been demonstrated that a truncated version of the Nmd3 protein lacking its last 100 amino acids, which includes its NES but not its nuclear localization sequence (NLS), is able to bind pre-60S r-particles that remain trapped in the nucle(ol)us [107]. Dissociation of Nmd3 from cytoplasmic pre-60S r-particles is imperative for $r$-subunit joining and translation initiation [109]. Moreover, this reaction involves the activity of the cytoplasmic GTPase Lsg1/Kre35 and the assembly of L10 (uL16), which seems to stably lock into its final position on cytoplasmic pre-60S r-particles concomitantly to the removal of $\mathrm{Nmd} 3$ $[110,111]$. In line with this model, distinct mutations in LSG1 or RPL10 or depletion of L10 cause a retention of $\mathrm{Nmd3}$ on cytoplasmic pre-60S r-particles and are synthetically lethal with specific $n m d 3$ mutants [111], while overexpression of wild-type $\mathrm{Nmd} 3$ or the presence of mutated $\mathrm{Nmd} 3$ versions with reduced affinity for pre-60S r-particles suppress the growth defect and the failure to recycle $\mathrm{Nmd} 3$ in $/ s g 1$ and/or rp/10 mutants [111, 112].

Cryo-EM analyses of purified mature LSUs harbouring in vitro-reconstituted $\mathrm{MBP}$-tagged $\mathrm{Nmd3}$ or of native Nmd3-purified pre-60S r-particles have shown that Nmd3 binds to the intersubunit face of pre-60S r-particles spanning from the L1 (uL1) stalk to the position where Tif6 binds (see later), going through the E- and P-sites (Figure 5C) and contacting helices $\mathrm{H} 38$ (also known as the A-site finger), $\mathrm{H} 65$ and $\mathrm{H} 95$, in addition to the sarcin/ricin loop (SRL) in $25 S$ rRNA $[43,113,114]$. These findings are consistent with the Nmd3-binding sites detected by the CRAC method (Figure 5A) [115]. The association of Nmd3 to pre$60 \mathrm{~S} r$-particles seems to be very dynamic and, in unison with the L1-stalk, it could adopt several states [114]. Most, if not all, of these states are incompatible with the simultaneous presence of the Sdo1•Efl1 complex in the $r$ particles (also discussed later) $[114,116]$ or r-protein $L 40$ (eL40), which, as L10, also assembles in the cytoplasm [117]. In agreement, purified pre-60S r-particles purified via TAP-tagged Nmd3 clearly lack some r-proteins, among them L10, L40, L12 (uL11) and L41 (eL41) [43]. Thus, due to the fact that mature LSUs contain r-proteins L10 and L40, it is expected that the position of $\mathrm{Nmd} 3$ in the in vitro reconstituted LSUs may not fully correspond with the one it adopt within native pre-60S r-particles $[43,114]$ (see also Figure $5 \mathrm{C}$ ). Moreover, as a corollary of the steric clash between Nmd3 and Sdo1・Efl1, it could be deduced that Lsg1 might not bind to the GTPase-associated centre (GAC) on pre-60S r-particles; indeed, the Hurt laboratory has suggested that Lsg1 could contact pre-60S r-particles also at the interface face of LSU but close to helix $\mathrm{H} 69$ of 25 S rRNA 
B

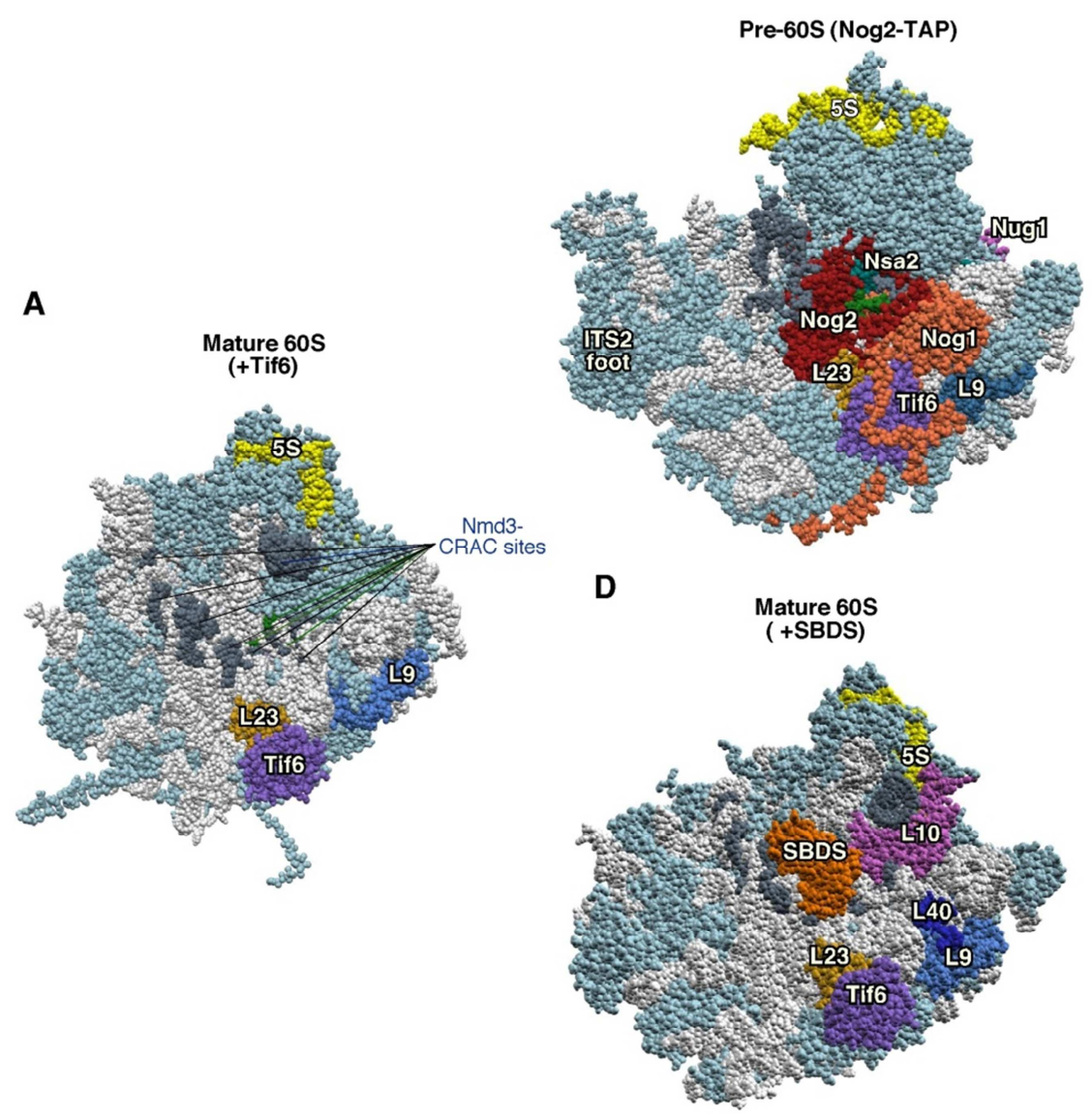

C

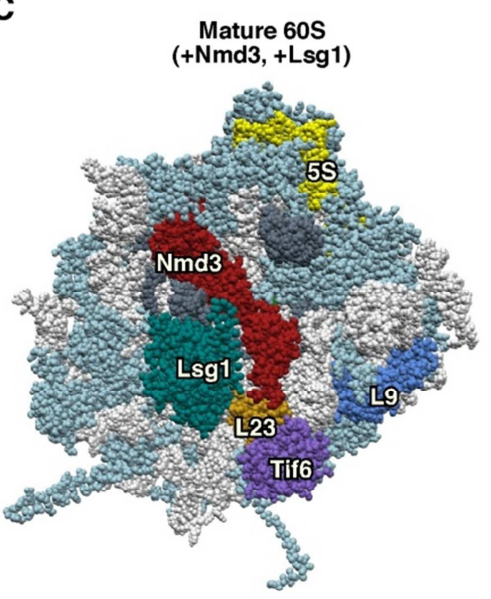

E

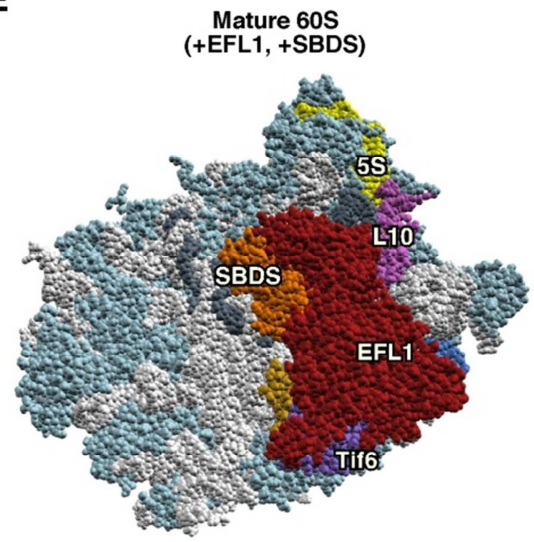

Figure 5: Interactions at the interface side of cytoplasmic pre-60S ribosomal particles. (A) The Nmd3-binding sites, identified by CRAC at $25 \mathrm{~S}$ rRNA helices H38, H69-69 and H89-90 [115], have been highlighted in dark grey in a reconstituted 60S r-subunit (PDB ID:5T62; [114]). The CRAC sites common for Nmd3, Dbp10 and Nug1 [115, 118] are labelled in green. (B) Position of Nog2 (red), Nsa2 (blue), Nog1 (gold) and Nug1 (pink) in the early pre-60S r-particle purified with Nog2-TAP (PDB ID: 3JCT; [40]). Note that only a very small portion of Nug1 has been resolved in this particle. (C) Position of Nmd3 and Lsg1 in a reconstituted 60S r-subunit (PDB ID:5T62; [114]). Note that only the region of Nmd3 comprised between residues 46 and 388 of 518 in total is shown. The N-terminal end of Nmd3 approaches to Tif6 while the C-terminal end contact L1. In A-C, the position of Tif6 (purple) is shown. The locations of the $5 \mathrm{~S} \mathrm{rRNA} \mathrm{(yellow)} \mathrm{and} \mathrm{that} \mathrm{of} \mathrm{the} \mathrm{L23} \mathrm{(gold)} \mathrm{and} \mathrm{L9} \mathrm{r-proteins} \mathrm{(royal} \mathrm{blue)} \mathrm{have} \mathrm{also} \mathrm{been}$ highlighted. (D) Position of SBDS (dark gold) in the 60S r-subunit. The structure of a pre-60S r-particle from Dyctiostelium discoideum containing Tif6 and reconstituted with human SBDS and EFL1 (PDB ID: 5ANB; [116]) was superimposed on the structure of yeast 60S r-subunit (PDB ID: 5APN; [68]); then, all common proteins from D. discoideium were removed from the model. L9 (royal blue), L40 (navy blue), L10 (violet), L23 (gold) and $5 S$ rRNA (yellow) were highlighted. Note that SBDS is shown in its open conformation state. (E) As D, but additionally showing EFL1 (red) on top of Tif6. In all figures, the Nmd3-CRAC sites shown in A (dark grey) were also highlighted, the rest of rRNAs are coloured in pale blue and the rest of $r$ proteins and/or factors in light cornflower blue.

and the P-site [42]. These suggestions have been later confirmed by cryo-EM analysis of native or reconstituted Nmd3-containing particles [43, 114].

These cryo-EM studies fully explain why r-subunit joining and translation are not possible as long as $\mathrm{Nmd} 3$ is not released from pre-60S r-particles. Moreover, they could also reveal that the C-terminal domain of $\mathrm{Nmd3}$ adopts a structure that mimics that of translation elongation factor elF5A and binds similarly as elF5A to the E-site of pre-60S $r$ subunits [114]. Importantly, these studies also revealed that most of the Nmd3-binding sites overlap with those of the GTPase Nog2 [115], which have been determined by cryo-EM [40]. In agreement, purification of pre-60S $r$ particles indicates that $\mathrm{Nog} 2$ and $\mathrm{Nmd} 3$ are not simultane- ously present on the same pre-60S r-particles (Figure 5). Different experimental approaches indicate that the binding of Nog2 to early pre-60S r-particles precedes that of Nmd3 (e.g. [40]), and consistently, the depletion of Nog2 causes the premature binding of $\mathrm{Nmd} 3$ to these particles [115].

Altogether, these experiments indicate that Nog2 is the placeholder factor of $\mathrm{Nmd3}$, which acts by blocking the premature recruitment of the latter and, therefore, providing the time frame necessary for pre-60S r-particles to acquire their export competence. Interestingly, this activity seems to be coupled to that of other trans-acting factors, such as the AAA-ATPase Rea1 and its substrate Rsa4, in a way where stable binding of Rea1 and Rsa4 to pre-60S r- 
particles requires Nog2, and the release of Nog2 from pre$60 \mathrm{~S}$ r-particles requires not only its GTPase activity, but also the ATPase activity of Rea1 [115]. Nmd3, on the other hand, supervises structurally and functionally the flexibility of the L1-stalk and the correct conformation of the E- and P-sites of LSUs; together with other ribosome assembly factors, such as Tif6, Nmd3 also impedes premature association of pre-60S r-particles with SSUs.

\section{Ef|1•Sdo1 probes critical functional sites on pre-60S r- particles during LSU maturation}

Efl1/Ria1 and Sdo1 (yeast SBDS orthologue) are quasiessential trans-acting factors required for cytoplasmic LSU maturation [19, 119, 120]. Efl1 is a cytoplasmic GTPase composed of five structural domains; it is highly homologous to the translation elongation eEF2 factor, which is responsible for the translocation reaction of the ribosome following each round of polypeptide elongation $[1,19,119]$ In turn, Sdo1 is a very flexible protein, formed by three domains, which resembles bacterial ribosome recycling factor RRF $[116,121]$. Both proteins are functionally related to each other, Tif6 and r-protein L10 [19, 119, 121, 122]. Based on the structural similarity, it has been suggested that Efl1 interacts with pre-60S r-particles in a very similar manner as eEF2 does with 805 ribosomes; recruitment of Efl1 needs the presence of $\mathrm{PO}$, the largest component of the ribosomal P0/P1/P2 stalk (uL10/P1/P2) [55] and the Pstalk base, the r-protein L12 (uL11), on the pre-60S $r$ particles [48]; then, Sdo1-stimulated GTP hydrolysis might trigger a sort of translocation reaction that facilitates the dissociation and recycling of Tif6 (see [116, 120-122], and references therein).

Recently, cryo-EM analyses of reconstituted pre-60S $r$ particles containing or lacking endogenous Tif6 from Dictyostelium discoideum and harbouring both human EFL1 and SBDS have allowed building models that deduce the precise interaction of these three factors with pre-60S $r$ particles (Figure 5). These models also provide a molecular scenario to understand how the release of Tif6 from these particles occurs [116]. In this possible scenario: (i) first, SBDS is recruited to Tif6-containing late cytoplasmic pre$60 S$ r-particles, in which the assembly of L10 and the Pstalk have already occurred. Tif6 is at its canonical position on the LSU interface bound to the C-terminal part of L23 (uL14) in the proximity of the SRL and the N-terminus of $\mathrm{L} 24$, thereby inhibiting $r$-subunit joining and, thus, preventing pre-60S r-particles from prematurely engaging in translation [47, 123]; the three domains of SBDS adopt a "closed conformation": domain I occupies the P-site of LSU and is in contact with a flexible loop of L10 (named P-site loop) [122], components of the PTC and the entrance of the PET; domain II mediates binding of SBDS to 25S rRNA, and domain III, which structurally resembles domain $V$ of Efl1, contacts the SRL and the neighbouring base of the P-stalk [116]. (ii) Second, EFL1 in its GTP-bound form binds the nascent GAC from the pre-60S r-particles, while contacting both Tif6 and SBDS, and r-proteins P0 and L12. Interestingly, the binding of domain III of SBDS on pre-60S r-particles is mutually exclusive with that of domain $\mathrm{V}$ of EFL1, thus, it seems that, upon EFL1 recruitment, SBDS undergoes a switch to reposition itself in a "open conformation" where its domain II rotates $60^{\circ}$ relative to its domain I and, especially, its domain III rotates $180^{\circ}$ away from the base of the P-stalk [116]. (iii) These changes are supposed to accommodate EFL1 into the GAC leading to slight conformational adjustments that result in a more extensive binding of domain I of EFL1 to the SRL in the pre-60S r-particles; this new SRL-bound conformation overlaps with part of the Tif6-binding site and it is supposed to facilitate the release of Tif6 from these particles. (iv) The accommodated state of EFL1 also seems to stimulate the GTP hydrolysis of the factor, which causes another conformational change of EFL1 that destabilizes its interaction with pre-60S $r$ particles and that of SBDS; as a consequence, both SBDS and GDP-bound EFL1 dissociate from these Tif6-lacking pre-60S r-particles. Still, the exact role of GTP hydrolysis for Tif6 eviction remains to be elucidated [116].

Together, these findings indicate that Efl 1 and Sdo1, as other above-mentioned examples, are factors with decisive roles in timing LSU maturation. Sdo1 examines the structure and function of important sites of LSUs, such as the PTC, the P-site and the entrance of the PET. Sdo1 seems to verify the assembly of L10. In turn, Efl1 proofreads the integrity of the GAC in LSUs, once the P-stalk has been properly assembled. Any delay or defect in the evaluation of all these sites would have as a consequence the inefficient release of Tif6 from pre-60S r-particles, thus, preventing translation by these apparently aberrant particles. Warren and co-workers assume that the Efl1•Sdo1-dependent maturation reaction might occur downstream of the assembly of L10 and the release of $\mathrm{Nmd} 3([107,108,111]$; for a review, see [17]). This scenario differs from the one previously and currently reported by the Johnson laboratory, claiming that the release of Tif6 occurs prior to $[18,55]$ or could be coupled with the Lsg1-mediated release of Nmd3 [114], respectively. Future experiments are clearly required to clarify these issues.

\section{Nog1, Rei1 and Reh1 are plugs that block the nascent} polypeptide exit tunnel of LSUs

As previously mentioned, cryo-EM has allowed to deduce that Nog1 is the placeholder of Rei1 (for a review, see [124]). Notably, both Nog1 and Rei1 enter the tunnel from the exit side with their $\mathrm{C}$-terminal ends oriented to the PTC $[40,68]$ (Figure $6 \mathrm{~A}$ and $\mathrm{B}$ ). This $\mathrm{N}$ - to $\mathrm{C}$-terminal orientation resembles that of the nascent polypeptide chain during translation. Nog1 is replaced by Rei1 in the cytoplasm, however, the precise mechanism of this exchange is still elusive [55-58]. Another protein, SBDS (Sdo1), is able to bind the exit tunnel, but in this case, the interaction takes place only via a very short extension, involving tunnel insertion of the N-terminal end of Sdo1, nearby the PTC [116]. In a recent review, B. J. Greber has nicely modelled how SBDS and Rei1 interact without overlapping in the tunnel in an arrangement where the $\mathrm{N}$-terminal end of SBDS is positioned close to the C-terminal end of Rei1, a circumstance that could not occur in the case of SBDS and Nog1 [124]. In this manner, the entire tunnel is filled, thus, 
A

Pre-60S (Nog2-TAP)

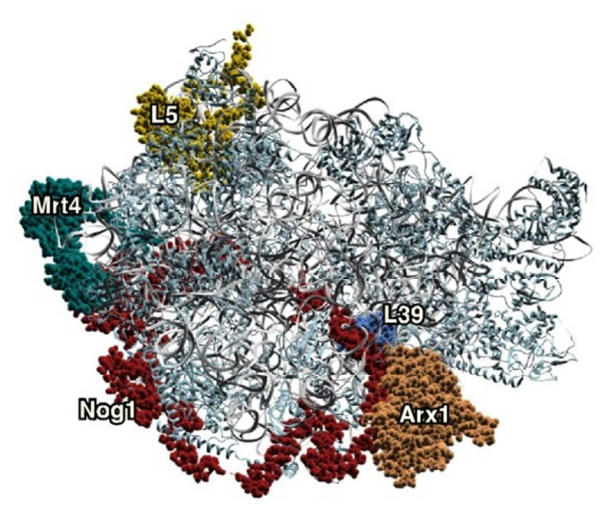

B

Mature 60S
(+Rei1 +Arx1 +Alb1)

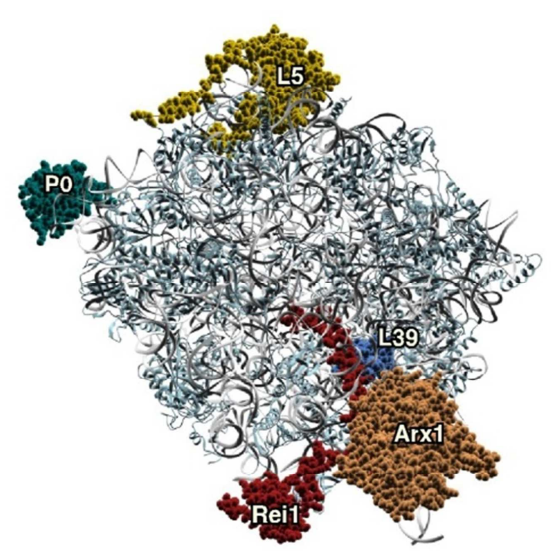

C

Pre-60S (Nmd3-TAP)

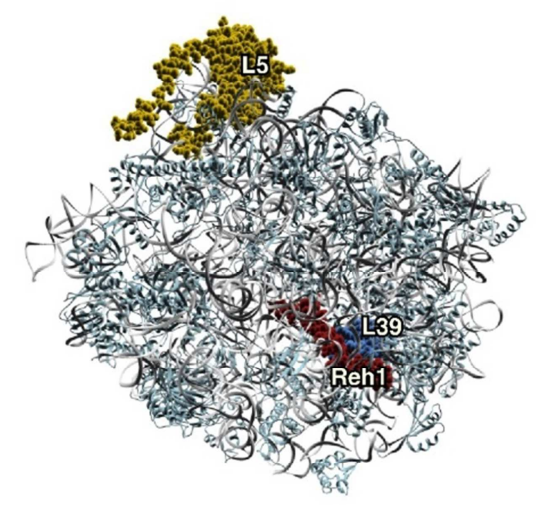

Figure 6: Interactions at the polypeptide exit tunnel of pre-60S ribosomal particles. (A) Position of Nog1 (red) and Arx1 (dark gold) in the early pre-60S r-particle purified with Nog2-TAP (PDB ID: 3JCT; [40]). Note that the C-terminal part of Nog1 enters the tunnel. The location of Mrt4 (cyan) is also shown. (B) Position of Rei1 (red) and Arx1 (dark gold) in a reconstituted 60S r-subunit (PDB ID: 5APN; [68]). Note that only the middle part (141-261) and the C-terminal end (300-393) of the protein is visualised. As above, the C-terminal part of Rei1 enters the tunnel. The location of P0 (cyan) is also shown. (C) Position of Reh1 (red) in the late/cytoplasmic pre-60S r-particles purified with Nmd3-TAP (PDB ID: 5H4P; [43]). Note that only the region of the protein occluding the tunnel could be visualised (amino acids 377-432 of 432). Particles are viewed from the solvent side. For orientation, the locations of L5 (yellow) and L39 (blue) have been highlighted. The rest of rRNAs are coloured in pale blue and the rest of r-proteins and/or factors in light cornflower blue.

providing a situation that could allow the complete functional proofreading of the integrity of this ribosomal site in cytoplasmic pre-60S r-particles.

Recently, the C-terminal helix of Reh1, which is highly homologous to that of Rei1 both at the level of sequence and structure, has also been found inserted in the PET of cytoplasmic pre-60S r-particles. The orientation of the Cterminal tail of Reh1 inside the tunnel is similar to that of the C-terminal extension of Rei1 (Figure 6C) [43]. It seems that Reh1 binds downstream of Rei1 during the maturation of these particles, as suggested by the fact that Reh1containing pre-60S r-particles lacks the Arx $1 \bullet A l b 1$ complex [63].

\section{Nug1 and Dbp10: more overlapping interactions}

The conformation of the nascent PTC is also verified by different factors during LSU maturation, which are expected to act sequentially on this site (Figure 5). First, distinct residues of the PTC are known to be subjected to pseudouridylation and 2'-O-methylation by specific snoRNPs (for an example, see [125]), or base methylation by the site-specific methylases Spb1 and Nop2 [126, 127]. These snoRNPs and methylases seem to associate with and act on early pre-60S r-particles [53, 128]. Second, also the trans-acting factor Nsa2 [129] has been shown to bind to rRNA near the immature PTC, most specifically to positions close to the base of helix H89 in 25S rRNA [130], where it interacts with Nog1 and Nog2 [40]. Interestingly, Nsa2 associates with pre-60S r-particles only after formation of 27SB pre-rRNAs $[53,131]$ and this association is dependent on the presence of other LSU maturation factors, including Nop2, Dbp10 and Nog1, on pre-60S r-particles [53]. Third, it has been also shown that the RNA helicase Dbp10 binds around the base of helix $\mathrm{H} 89$ [118]. These sites partially overlap with those of Nsa2, Nmd3, and of GTPases Nug1 and Nog2 [115, 118, 130]. Indeed, Nug1 and Dbp10 functionally interact with each other [132]. Moreover, the association of Dbp10 with pre-60S r-particles is dependent on the presence of Nug1 [118].

All these findings, together with the above described binding sites of Nmd3, Nog1, Nog2 or Sdo1, as well as the assembly position of r-proteins such as L10, provides an idea of the, still to be unveiled, complex arrangement of sequential actions and interactions of factors during the maturation of strategically relevant, functional sites of $r$ subunits (for a review, see also [124]).

\section{Arx1 versus translation-associated factors that bind the ribosome exit tunnel.}

Arx1 is a non-essential trans-acting factor that has the capability to bind to FG-repeat nucleoporins, thereby, functioning as a RanGTP-independent export factor of pre-60S r-particles [133-135]. The human orthologue of yeast Arx1, EBP1, folds as a MetAP, an enzyme removing the $\mathrm{N}$ terminal methionine from nascent polypeptides as they emerge from the exit tunnel of the ribosome [133]. Given the close homology between EBP1 and Arx1, it was deduced that Arx1 also conserves the MetAP core fold. However, both EBP1 and Arx1 lack MetAP activity, as the critical residues of the methionine-binding pocket are different [133]. The interpretation of the cryo-EM structures of either purified pre-60S r-particles containing Arx1 [70] or in vitro reconstituted 605 r-subunits complexed with Arx1 [69] is in full agreement with this deduction. In these mod- 
els, Arx1 binds nearby to the outside part of the PET (Figures $6 A$ and $6 B$ ) $[69,70]$ in a position that seems to overlap with the one of MetAPs [136]. Consistently with this, the rRNA residues contacted by Arx1, which were identified by CRAC analysis, are clustering at the outside of the ribosome exit tunnel [70]. Moreover, either the addition of a GFP-tag to r-proteins L25 (uL23) or L35 (uL29), which both surround the outside of the PET, or the depletion of L35 significantly reduce the binding of Arx1 to pre-60S $r$ particles [67, 137].

In conclusion, these findings indicate that Arx1 could act as a structural proofreader of the correct formation of the solvent-side part of the exit tunnel [138]. The inefficient recruitment of Arx1 to eventually aberrant pre-60S rparticles may impair export of these particles to the cytoplasm, leading to their transient accumulation in the nucleus and inducing their rapid turnover. Arx1 may also represent a placeholder for those ribosome-associated factors that bind the outside of the tunnel during translation, such as the ribosome-associated complex, the nascent polypeptide-associated complex, the chaperones Ssb1/Ssb2 or the signal recognition particle (for a review see, [139]). This activity could prevent the premature recruitment of all these factors. The presence of Arx1 at its binding site also impairs the association of pre-60S r-particles with the endoplasmic reticulum-translocon complex, which acts as a channel to deliver nascent proteins to the lumen of the endoplasmic reticulum (e.g. see [140]).

\section{PARAPHERNALIA FOR THE NUCLEAR IMPORT AND ASSEMBLY OF RIBOSOMAL PROTEINS}

Most newly synthesized $r$-proteins need to be transported to the nucle(ol)us to reach their assembly sites in the preribosomal particles. The specific interactions of the $r$ proteins with the general import factors, mostly $\beta$ karyopherins [141, 142], are normally mutually exclusive with their interactions with the rRNAs. This feature also applies for the interactions that selected $r$-proteins undergo with specific factors that help their import or assembly, also referred as dedicated chaperones and escortins [143145]. In addition to help import or assembly, $\beta$ karyopherins, dedicated chaperones and escortins prevent the aggregation of $r$-proteins, which are prone to aggregate since they contain highly basic and intrinsically disordered extensions [146]; these factors also impede $r$ proteins to either be degraded [147] or inappropriately interact with other cellular RNAs prior to their assembly into pre-ribosomal particles [146].

In a recent report, it has been shown that most, if not all, NLSs of yeast $r$-proteins reside within long non-globular extensions of the proteins. These extensions thread across the surface of the r-subunits making extensive contacts with the rRNAs or penetrate into the interior of the $r$ subunits intertwining with and stabilizing rRNAs [141]. Kap123 is the RanGTP-dependent $\beta$-karyopherin in charge of recognising the NLSs of most r-proteins [142], although other $\beta$-karyopherins, such as Kap104, Kap108 or Kap121 $[143,148,149]$, and even the importin- $\alpha$ Kap60 [150] have been reported to bind the NLSs of specific r-proteins. To our knowledge, no structural data of any r-protein bound to a karyopherin are available at atomic resolution, except for the recent determination of the crystal structure of Kap104 in complex with the PY-NLS of L4 (uL4) [149]; here, the residues of the PY-NLS of L4 engages the concave surface on the structure of Kap104, similarly as other importins recognize their selected NLSs [145].

So far, seven specific systems, composed of a dedicated chaperone or an escortin and an r-protein, have been reported in yeast, a list that may still be far from being completed: $\quad A c l 4 \bullet L 4, \quad B c p 1 \bullet L 23, \quad \operatorname{Rrb} 1 \bullet L 3, \quad S q t 1 \bullet L 10$, Syo1 $\bullet$ L5 $\bullet$ L11, Tsr2 $\bullet S 26$ and Yar1 $\bullet S 3$ (for a review, see [151]). For most of them, the binding sites of the chaperone on the respective $r$-protein have been mapped, and for several of them structural information is also available. From all these studies, it could also be concluded that the mode of interaction of the respective r-proteins with their specific chaperone partners is highly similar and mutually exclusive with that observed for these $r$-proteins when assembled into the pre-ribosomal particles. Interestingly, most of these chaperones capture co-translational their targets by, in most cases, interacting with their very $\mathrm{N}$ terminal ends, a property that minimizes the risk of $r$ protein aggregation [152].

\section{Acl4 and $\mathrm{L} 4$}

Acl4 is a non-essential tetratricopeptide repeat-like (TPRlike) factor, which has been identified as the specific dedicated chaperone of free $L 4$ (uL4) $[153,154]$. Acl 4 constitutes one exception to the principle of co-translationally recruitment through the $\mathrm{N}$-terminal end of the $\mathrm{r}$-protein partner since, although it is true that Acl 4 is recruited to nascent $L 4$, it directly interacts with the long internal loop of the r-protein (amino acids 43-114) [149, 153, 154]. Additionally, the eukaryote-specific C-terminal extension of L4 harbours a PY-NLS, which binds specifically to another Acl4 molecule that is later replaced by the karyopherin Kap104 $[149,153,154]$. Importantly, both the internal loop and the C-terminal tail of $L 4$ occupy strategic positions in mature LSUs $[44,47]$; the internal loop penetrates into the core of the LSU in the direction to the PTC, forming a narrow constriction within the exit tunnel where it even forms some contacts with various residues of the nascent protein chains [155]; the C-terminal tail of L4 threads a long tour across the solvent-exposed interface of the LSU in direction of the P-stalk, approaching and making numerous contacts with other r-proteins, such as L18 (eL18), L7 (uL30), L20 (eL20) and L21 (eL21) and rRNA sequences, including the expansion segments ES15 and ES7 of the 25S rRNA [44, 156]. Recently, the crystal structure of $L 4$, excluding its Cterminal extension, in complex with Acl4 has been resolved at atomic resolution [149]. In this complex, the majority of the internal loop of $L 4$ is bound by the concave surface of $\mathrm{Acl} 4$, an interaction that is totally mutually exclusive with that this region of L4 forms within the LSUs [149].

It has been shown that Acl 4 actively travels as a trimeric Kap104•L4•Acl4 from the cytoplasm to the nucle(ol)us where the assembly of the r-protein in early pre-60S $r$ - 
particles takes place $[79,152]$. As for other specific chaperones, Acl4 does not significantly associate with pre-60S rparticles $[153,154]$, but is expected to facilitate the assembly of L4 into these particles; how this process mechanistically occurs is currently unclear.

\section{Rrb1 and L3}

Rrb1 is WD-repeat protein predicted to form a $\beta$-propeller structure and involved in LSU maturation $[157,158]$. The function of Rrb1 in LSU biogenesis is linked to that of the $r$ protein L3 (uL3) [157, 158]. Indeed, the Kressler laboratory has nicely demonstrated that Rrb1 captures nascent L3 in a co-translational manner via its interaction with the first 15 amino acids of the r-protein [152]. Following this cytoplasmic recognition, Rrb1 then accompanies L3 to its assembly site on early nucleolar pre-60S r-particles [78, 152, 157]. Unfortunately, no structural data have so far been reported for this interaction, although it has been speculated to occur in a similar manner as that between Sqt1, another WD-repeat protein, and its specific partner, the $r$ protein L10 (see below) [151]. Despite this issue, it is clear that the interaction of L3 with Rrb1 is mutually exclusive with the ribosomal interaction of $\mathrm{L} 3$. In this sense, the $\mathrm{N}$ terminal end of $\mathrm{L} 3$, which is recognized by Rrb1, penetrates deep into the core of the LSU where it reaches towards the PTC, nested between helices H90 and H92 of 25 S rRNA [44, 159].
The assembly path of L3 is totally unknown. Given the specific interaction of both L 3 and Rrb1 with the members of the so-called the Dbp6-containing subcomplex (see [160]), it is expected that the transfer of L3 from Rrb1 to its assembly site in early pre-60S r-particles could be facilitated by this subcomplex.

\section{Sqt1 and L10}

The essential WD-repeat protein Sqt1 is a dedicated chaperone that has been proposed to facilitate the assembly of L10 (uL16) into cytoplasmic pre-60S r-particles [110, 152, 161]. Two decades ago, Sqt1 was identified as a high-copy suppressor of selected $r p / 10$ mutants [161]. Additionally, it has been reported that Sqt1 interacts with L10 by the twohybrid system [161], by co-immunoprecipitation [110, 152] or by an in vitro binding assay [152]. Sqt1 recognizes the Nterminal part of $L 10[110,152,162]$ and, as described for Acl4 and Rrb1, the chaperone is already recruited to the nascent $L 10$ as it is translated from its mRNA [152]. The complex between the WD-repeat propeller-like domain of Sqt1 and the N-terminal part of $\mathrm{L} 10$ has been cocrystallized and structurally resolved to atomic resolution [152]. This analysis shows how the very basic $\mathrm{N}$-terminal end of L10, which forms an $\alpha$-helix, is accommodated by the negatively charged top surface of the propeller [152]. Importantly, this study also revealed that the conformation of the corresponding $\mathrm{N}$-terminal residues of L10 bound to
A

$5 S$ rRNP•60S

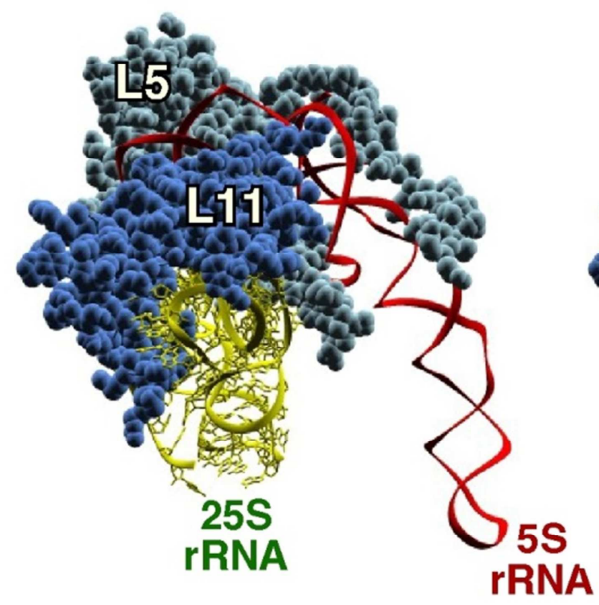

B

$5 S$ rRNP•Syo1

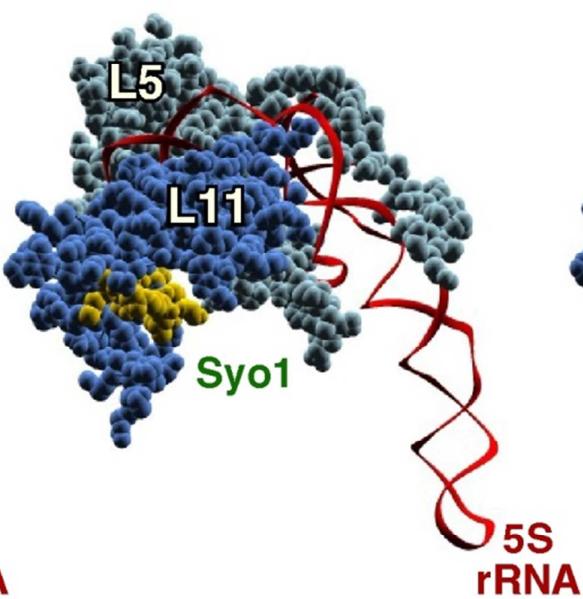

5S rRNP•MDM2

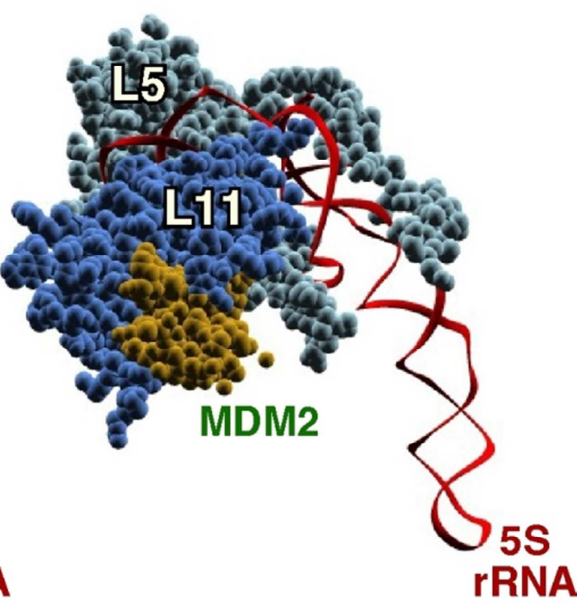

Figure 7: The helix H84 in the 60S r-subunit, the symportin Syo1 and the p53 regulator MDM2 share the same binding site on L11 r-protein. (A) Structure of the 5S RNP as is assembled in the mature 60S r-subunit bound to 25S rRNA (nucleotides 2651-2750 comprising helices H83, H84, H85 and H86). (B) Interaction of a specific region of Syo1, called Syo1-HS (amino acids 328-384), with L11 in the context of the 5S RNP. (C) Structure of the 5S RNP bound to a distinct fragment of MDM2 (amino acids 293-334). The fragment of 25S rRNA is coloured in yellow, that of Syo1 in light gold, and that of MDM2 in dark gold; 5S rRNA in highlighted in red, L5 in cyan and L11 in blue. PDB ID: The 5S RNP bound to $25 \mathrm{~S}$ rRNA was taken from 3U5I and 3U5H [44]; Syo1-HS fragment was extracted from 5AFF [163] after superimposing the structure shown here with that present in the PDB file 4GMN [144]; MDM2 fragment was taken from 4XXP [164] after superimposing the structure shown in this file with that of L11 shown in A. 
Sqt1 is not compatible, hence mutually exclusive, with the positioning of these residues, which interact with helix $\mathrm{H} 89$ of $25 \mathrm{~S}$ rRNA, in the mature LSU. This information, together with all the genetic and biochemical data available for L10 and Sqt1, as well as those factors functionally related to L10, such as Nmd3, Sdo1 or Efl1, is very relevant to unravel the molecular dynamics of the delivery of L10 from Sqt1 to cytoplasmic pre-60S r-particles, which is still not fully understood (see [152] for further discussion of this issue).

\section{Syo 1 and L5 and L11}

The symportin Syo1 is an exceptional factor since it is the only described case of a chaperone dedicated to the synchronous co-import of two conserved r-proteins to the nucle(ol)us: L5 (uL18) and L11 (uL5), which are the two protein components of the 5S rRNP structure of LSUs [144]. The crystal structure of $C$. thermophilum Syo1 complexes reconstituted with L5, L11 and/or 5S rRNA have been reported $[144,163]$; Syo1 is an open $\alpha$-solenoid comprised of four consecutive ARM-repeats followed by six HEATrepeats; notably, the acidic loop, connecting the two first HEAT repeats long, harbours a critical helical segment, known as Syo1-HS, for function. While the ARM-repeat domain is not involved in cargo binding, the HEAT-repeat domain can simultaneously accommodate both L5 and L11 on opposite sides [144]. More specifically, the eukaryotespecific $\mathrm{N}$-terminal end of $\mathrm{L} 5$ binds a groove formed at the inner surface by the four first HEAT-repeats of Syo1 [144], while L11, which adopts a hand-like shape, primarily interacts via the internal concave $\beta$-sheets from its "palm" with the Syo1-HS segment; other contacts between Syo1 and L11 are made between a particular loop located at the "knuckles" of the r-protein and the outer side of Syo1's HEAT repeats 1 to 3 (for details, see [163]). Strikingly, the $\mathrm{N}$-terminal end of $\mathrm{L} 5$ also contributes to $5 \mathrm{~S}$ rRNA binding $[44,47]$ and the binding of L11 to the Syo1-HS is basically identical to and mutually exclusive with the interaction with the helix $\mathrm{H} 84$ of the $25 \mathrm{~S}$ rRNA (Figures 7A and 7B) in both pre-60S r-particles [41] and mature LSUs [44, 47].

The intensive work performed on Syo1, together with the significant amount of genetic, biochemical and structural data available on the formation of the 5S RNP complex, has allowed to establish the following model for the assembly of this complex into early pre-60S r-particles: (i) first, Syo 1 captures nascent $L 5$, in this manner, preventing L5 to misfold and aggregate [152]. Binding of L11 to Syo1 also apparently occurs in the cytoplasm but not in a cotranslational manner [144, 154]. (ii) The trimeric Syo1 $\bullet 5 \cdot L 11$ complex is then recognized, via the $\mathrm{N}$ terminal PY-NLS of Syo1, by the importin Kap104, and escorted to the site of assembly in the nucle(ol)us [144]. (iii) Upon nuclear arrival, RanGTP promotes the release of Kap104 and, concomitantly, the 5S rRNA likely binds to the trimeric complex [144]. Structural data suggest that binding of $5 \mathrm{~S}$ rRNA induces a change in the conformation of both $r$-proteins within the Syo $1 \bullet L 5 \bullet L 11$ complex [163]. (iv) Now, the pre-5S RNP is likely ready for incorporation into early pre-60S $r$-particles $[41,165]$, a process that is facilitated by the assembly factors Rpf2 and Rrs1 [166]. Struc- tural and functional analyses strongly suggest that the Rpf2・Rrs1 complex can bind the Syo $1 \bullet 5 S$ rRNA $\bullet 5 \cdot L 11$ complex, by this way recruiting it to nascent pre-ribosomal particles [167-169]; however, Syo1 is never found associated to pre-ribosomal particles, thus, the interaction of Rpf2•Rrs1 complex with 5S RNP in the context of pre-60S $r$ particles must somehow induce the recycling of the symportin [167, 169]. Interestingly, the recruitment of 5S RNP to particles includes the docking of L11 to helix H84 of $25 \mathrm{~S}$ rRNA, while keeping the rest of the RNP in an almost $180^{\circ}$ rotated configuration that is different from the one adopted in mature 605 r-subunits $[41,167,169]$. Since the Rpf2•Rrs1 complex (and Rsa4) interacts with the 5S RNP in this initial conformation but is unable to do it in the rotated, final conformation in mature LSUs, it has been suggested that the Rfp $2 \bullet R r s 1$ complex defines the timing for assembly of 5S RNP into distinct pre-60S pre-ribosomal particles, thereby exerting a quality control surveillance for this important step during the formation of LSUs $[130,169]$.

\section{Escortins of r-proteins: Tsr2 for S26, and Bcp1 for L23}

Another factor involved in the correct assembly of a specific r-protein is Tsr2. This factor was first identified as a nonessential protein required for $20 \mathrm{~S}$ pre-rRNA maturation, and hence for SSU biogenesis [170]. More specifically, Tsr2 has been described to be required for cytoplasmic processing of $20 \mathrm{~S}$ pre-rRNA to mature $18 \mathrm{~S}$ rRNA [143]. This role of Tsr2 is clearly linked to its specific association with the r-protein S26 (eS26) $[143,170]$. Indeed, the Panse laboratory has shown that S26 binds directly to Tsr2 and is also required for cytoplasmic processing of $20 \mathrm{~S}$ pre-rRNA [143]. In clear contrast to other dedicated chaperones, Tsr2 only interacts with S26 once both proteins have been independently imported to the nucleus by selected karyopherins, such as Kap123 [143]. Biochemical evidence suggests that Tsr2 has the capacity to extract S26 from its karyopherin by a RanGTP-independent mechanism [143]. The Tsr2•S26 interaction impedes the aggregation of S26, while presumably permitting the escort of the r-protein to its correct assembly site on early $90 \mathrm{~S}$ pre-ribosomal particles. However, Tsr2 does not significantly interact with the nascent pre-ribosomal particles [143]. Due to this role, Tsr2 has been proposed to be an "escortin", which refers to any trans-acting factor able to release distinct $r$-proteins from their specific import systems and transfer them to the respective nascent pre-ribosomal particles where they assemble [143]. Further exciting experiments are clearly required to precisely understand how exactly Tsr2 and S26 interact with each other, and whether the Tsr2-bound state is mutually exclusive with that of S26 within the SSU. As discussed in the next section, $\mathrm{S} 26$ once dissociated from Tsr2, binds r-protein S14 to form a trimeric Fap7•S14•S26 complex ([171]; see later).

Bcp1 is an essential factor required for proper LSU maturation [172]. Recently, it has been suggested that Bcp1 works as a chaperone of L23 (uL14) [173]. More specifically, Bcp1 seems to work as an escortin to dissociate nuclear and non-ribosome bound L23 from any of the distinct karyopherins, Kap123 or Kap121, involved in its nu- 
clear-cytoplasmic import. In the nucleus, Bcp1 appears to guide the assembly of L23 in early pre-60S r-particles. Similarly to what has been previously reported for Tsr2, the exchange of $\mathrm{L} 23$ from its karyopherin to $\mathrm{Bcp} 1$ is also likely RanGTP-independent [173]. Unfortunately, as for Tsr2, neither structural data nor information on the binding site of Bcp1 on L23 has so far been reported. These issues are of special interest to understand how L23 is delivered from Bcp1 to pre-60S r-particles and to get insight into its relation with trans-acting factors such as Tif6, whose binding site mainly involves L23 [47, 116, 123].

\section{Yar1 and S3}

The last member of this as yet likely incomplete inventory of factors is the ankyrin-repeat factor Yar1, which directly interacts with the free r-protein S3 (US3) [174]. S3 consists of two well-defined globular domains, one $\mathrm{N}$-terminal and another C-terminal, preceded and followed by unstructured terminal extensions, respectively [175]. As expertly envisioned by D. Lycan about a decade ago [174], Yar1 works as a molecular chaperone to keep S3 protected from in vivo agregation [176].

Indeed, as described for many of the above dedicated chaperones, Yar1 directly interacts with free S3 by capturing its $\mathrm{N}$-terminal unstructured domain in a cotranslational manner [152]. Then, Yar1 accompanies S3 from the cytoplasm to the nucleus where it seems to assist the proper assembly of S3 into late pre-40S r-particles [176, 177]. The detailed characterization done for Yar1 has suggested the following scenario for the stable assembly of S3: (i) Yar1 co-translationally binds the $\mathrm{N}$-terminal domain of S3, particularly the $\mathrm{N}$-terminal $\alpha$-helix of S3, by a concave pocket that is formed by its four ankyrin repeats, while its fourth ankyrin-repeat mediates contacts with the C-terminal domain of S3 [152, 178, 179]. (ii) Importantly, it has been shown that cytoplasmic S3 dimerizes through its C-terminal globular domain in vitro and in vivo [178]. Curiously, in vivo, only one S3 N-terminal domain of the dimer is associated with Yar1, while the second one is bound by the importin- $\alpha$ Kap60/Srp1 [150]. Kap60 binds the very N-terminal monopartite NLS of S3, most likely through its major binding site for cargos, and then to importin- $\alpha$ Kap95 through its orthodoxal IBB domain [150]; the S3 NLS is located contiguous to the Yar1-binding site, however, it has been established that the binding of Yar1 and Kap60 to one N-terminal domain of S3 is mutually exclusive, suggesting that one Kap60 molecule rapidly replaces only one Yar1 protein in the (Yar1•S3)2 tetrameric complex [150]. (iii) This asymmetric $\mathrm{Kap} 95 \bullet \mathrm{Kap} 60 \bullet \mathrm{S} 3 \bullet \mathrm{S} 3 \bullet \mathrm{Yar} 1$ complex is actively imported into the nucleus, where rapid RanGTP-dependent dissociation of the importer system presumably occurs. Other importins have been proposed to be redundantly able to mediate the import of S3, including Kap123 [150]. (iv) How exactly the transition from Yar1-bound S3 to pre$40 \mathrm{~S}$ r-particle-bound S3 occurs is unclear; it is worth to mention that these two states are incompatible with each other, since the N-terminal domain of S3, which binds
Kap60 and Yar1, is in contact, with several neighbouring $r$ proteins, such as S10 (eS10), S20 (uS10) and S29 (uS14), and specific rRNA residues of the 18S rRNA helix $\mathrm{H} 41$ within mature SSUs $[44,91,141,178]$. Moreover, the $\mathrm{N}$ terminal domain of $\mathrm{S} 3$ in the $\mathrm{S} 3 \bullet$ Yar1 complex is rotated ca. $180^{\circ}$ relative to the $\mathrm{C}$-terminal domain compared to its configuration in mature SSUs, and therefore, away from its contact surfaces in SSUs. Additionally, the dimerization of S3 masks the SSU rRNA-binding site of the C-terminal domain [179]. Initial assembly of S3 within pre-40S $r$ particles is suspected to occur through the C-terminal domain. This fact implies that the second copy of S3 from the tetramer is released as soon as the assembly of one molecule of S3 initiates and likely assembles in parallel within another orphan pre-40S r-particle. Concomitantly, the remaining Yar1 molecule dissociates and is replaced by the trans-acting factor Ltv1, which seems to have higher affinity for S3 [179, 180]. Ltv1 and Yar1 have partially overlapping binding sites within the $\mathrm{N}$-terminal domain of S3, as demonstrated in vitro [179]. This result is consistent with the reported cryo-EM position of Ltv1 on pre-40S rparticles [21], and the sites of interaction of Ltv1, described by the CRAC methodology, within helices $\mathrm{H} 41$ and $\mathrm{H} 16$ of the $18 \mathrm{~S}$ rRNA, which are located at the head and the shoulder of the SSU, respectively [103]. Importantly, different evidence indicates that, although $\mathrm{S} 3$ is present in late pre-40S r-particles containing Ltv1, it is still not bound in its final conformation $[21,101,177,181]$. Thus, it can be extracted from these particles in the presence of a high salt concentration as a complex with Ltv1 and another 40S assembly factor, Enp1 [101]. Presumably, in pre-40S rparticles, S3 is associated via its C-terminal domain at a position close to its final binding site [180]. However, its $\mathrm{N}$ terminal domain is likely still in the rotated conformation it adopts when bound to Yar1, while it probably interacts similarly to the central region of Ltv1 $[179,182]$. (v) The stable incorporation of S3 must occur concomitantly to the release of Enp1 and Ltv1 from cytoplasmic pre-40S $r$ particles, which is dependent on the phosphorylation of at least Ltv1 at selected serine residues by the kinase Hrr25 (human casein kinase $\delta$ or $1 \varepsilon$ ) $[101,179,180]$. It has been postulated that the release of Ltv1 from pre-40S r-particles allows the $\mathrm{N}$-terminal domain of S3 to assemble into its mature binding site within the context of S10, S20 and S29 and $18 \mathrm{~S}$ rRNA residues of helix $\mathrm{H} 41[179,180]$. Indeed, the interaction of the C-terminal part of Ltv1 with pre-40S rparticles seems to be incompatible with the positioning of S3 and the presence of S10 in these particles [21, 103, 177]. The C-terminal domain of S3 also changes its conformation during the cytoplasmic maturation of SSUs so that it acquires its final position by stably interacting with $r$ proteins Asc1, which is the yeast orthologue of mammal RACK1, and S17 (eS17) (for experimental evidence, see references [21, 177]). More recently, refined cryo-EM analysis of late/cytoplasmic pre-40S r-particles confirmed that S3 is still not bound at its mature site in these precursor particles, however, this study has questioned this particular model, involving the repositioning of the globular N- and C-terminal domains of S3 [183]. 
Table 1. Examples of placeholding situations during the ribosome biogenesis pathway.

\begin{tabular}{|c|c|c|}
\hline Factor & Counterpart $^{1}$ & Reference \\
\hline \multicolumn{3}{|c|}{ Ribosomal-like proteins } \\
\hline Mrt4 & Mex67, P0 (uL10) & {$[20,45,46]$} \\
\hline Rlp24 & L24 (eL24) & [28] \\
\hline \multicolumn{3}{|c|}{ Trans-acting factors } \\
\hline Arx1 & EBP1, RAC, NAC, Ssb1/2, Sec61 complex & {$[69,70]$} \\
\hline Bms1 & Tsr1 (?) & {$[95,99]$} \\
\hline Dbp10 & Nmd3 & [118] \\
\hline Dim2 & Krr1 (?) & [189] \\
\hline Efl1 & EF-2 (Eft1/Eft2), Tif6 & {$[19,116]$} \\
\hline $\mathrm{Nmd3}$ & elF5A & {$[43,114]$} \\
\hline Nog1 & Rei1 & [40] \\
\hline Nog2/Nug2 & Nmd3 & {$[40,115]$} \\
\hline Nsa2 & Nmd3 & [40] \\
\hline Nug1 & $\mathrm{Nmd3}$ & [118] \\
\hline Rei1 & Reh1 & {$[43,68]$} \\
\hline Sdo1 & $\mathrm{Nmd3}$ & {$[114,116]$} \\
\hline Tsr1 & elF5B, Rio1 & {$[21,102]$} \\
\hline \multicolumn{3}{|c|}{ Dedicated chaperones and escortins } \\
\hline Acl4 & L4 (uL4) & {$[149,153,154]$} \\
\hline Bcp1 & L23 (uL14) & [173] \\
\hline Fap7 & S14 (uS11) & {$[171,184,185]$} \\
\hline Rrb1 & L3 (uL3) & {$[152,157,158]$} \\
\hline Sqt1 & L10 (uL16) & {$[152,162]$} \\
\hline Syo1 & L5 (uL18), L11 (uL5) & {$[144,163]$} \\
\hline Tsr2 & $\mathrm{S} 26(\mathrm{eS} 26)$ & {$[143,170,171]$} \\
\hline Yar1 & S3 (uS3) & {$[150,152,174,178,179]$} \\
\hline \multicolumn{3}{|c|}{ Box H/ACA snoRNP assembly factors } \\
\hline Naf1 & Gar1 & {$[188]$} \\
\hline Shq1 & H/ACA snoRNA & {$[186,187]$} \\
\hline
\end{tabular}

${ }^{1}$ A question mark indicates that the placeholder correspondence or the mutually exclusive interaction is suspected and has not been experimentally addressed.

Nevertheless, given the intrinsic dynamic nature of the maturation pathway of pre-ribosomal subunits, it could also be feasible that these precursors had already undergone many of the conformational changes, involving S3 and Ltv1 that have been suggested to occur. Moreover, whether these structural rearrangements end with the release of Enp1 and Ltv1 also needs further clarification.

\section{PLACEHOLDING BY MOLECULAR MIMICRY}

The term "Protein-RNA mimicry" applies to the capability of a protein (or a protein domain) to imitate the structure of a distinct domain of an RNA that normally binds to a different RNA or protein. The interaction of the latter molecule with either its natural partner or the mimicking protein is normally used as a control step for the correct function of the biological process where this molecule participates (for a review, see [190]). Few cases of molecular mimicry have been reported related to the ribosome; the most classical one groups those translation factors that mimic tRNAs and that bind to the ribosome similarly as tRNAs do [190]. The pathway of ribosome assembly has also taken advantage from using the protein-RNA mimicry concept. One of these examples, as already mentioned above, represents the Syo1-HS domain that imitates the $25 \mathrm{~S}$ rRNA helix $\mathrm{H} 84$, which seems to control the timing of assembly of L11 into pre-60S r-particles [163]. Strikingly, the same mimicry principle has been exploited for the activation of $p 53$ during the mammalian nucleolar stress response, which is triggered by sequestering the E3 ubiquitin ligase MDM2 through its complex with 5S RNP (reviewed in [191]). Thus, recent structural work has shown that, similarly to Syo1, a particular domain of MDM2 mimics the $25 \mathrm{~S}$ rRNA binding to L11 so that the interaction of 5S rRNP to MDM2 or to the LSU are also mutually exclusive (Figures 7A and 7C) [164]. In this section, we review on other biological relevant examples of mimicry on ribosome assembly (see also, [192]).

\section{Fap7 and S14}

Fap7 is a conserved essential ATPase required for SSU formation, more specifically for the cytoplasmic maturation of $20 S$ pre-rRNA to $18 S$ rRNA $[170,193]$. This function is 
linked to that of r-protein S14 (uS11) and coordinated with that of other late SSU assembly factors (namely, Dim1, Enp1, Nob1, Pno1 and Tsr1); indeed, mutations in the eukaryote-specific C-terminal tail of S14 block processing of $20 \mathrm{~S}$ pre-rRNA to mature $18 \mathrm{~S}$ rRNA similarly as the depletion of Fap7 [194]; moreover, those late SSU assembly factors persisted in $80 \mathrm{~S}$-like particles in the absence of Fap7 [104].

It has been shown that Fap7 directly and stably interacts with S14 in vitro $[193,195]$ and in vivo $[184,195]$ but only weakly or transiently with pre-40S r-particles [104, $171,193,195]$. The structure of the Fap7•S14 heterodimer has been solved. In this complex, the interaction of Fap7 with S14 blocks the RNA binding surface of S14 [184, 185], hence, the binding of Fap7 to S14 clearly competes with the positioning of S14 in its final conformation in mature or almost mature SSUs. Interestingly, the structural studies indicate that indeed, Fap7 acts as an RNA mimic, using protein side chains to reproduce specific contacts of the 18S rRNA with S14 [185]. Physiologically, this interaction which is regulated through the ATP binding and hydrolysis activities of Fap7 (for further details, see [184, 185]), suggests that Fap7 acts as a dedicated and enzymatic chaperone for S14. In this way, Fap7 might protect S14 from aggregation and/or nonspecific interaction with other RNAs and regulate the correct timing of S14 assembly into pre$40 \mathrm{~S}$ r-particles. In agreement with this, recombinant S14 from $E$. coli showed poor solubility unless it is co-expressed with Fap7 [185]; moreover, depletion of Fap7 causes a strong decrease in the in vivo protein levels of S14 in S. cerevisiae [171]. However, there is so far no evidence for co-translational capturing of S14 by Fap7 (discussed in [151]).

Although different scenarios have been proposed, the mode of S14 release from Fap7 and the mechanism of its concomitant incorporation into pre-40S $r$-subunits are still unclear $[171,184,185]$. Interestingly, the Panse laboratory has recently shown that the assembly of S14 and S26 into pre-40S r-particles is interdependent [171]. Fap7 promotes the formation of a ternary complex containing both S14 and S26 [171], which are neighbouring proteins that directly interact in the mature SSU [44, 91]. In the Fap7containing complex, S26 and S14 might interact with each other similarly as they do in the context of the mature SSU [171]. Thus, it was concluded that Fap7 is an example of a factor enabling nucleation a module of two r-proteins, which then assemble en bloc into relatively early pre-40S $r$ particles [171].

\section{Shq1, Naf1 and the formation of H/ACA snoRNPs}

Box H/ACA ribonucleoparticles (snoRNPs) are a family of small RNA-protein complexes conserved in archaea and eukaryotes that convert uridine into pseudouridine at specific sites within rRNAs and snRNAs, mediate early prerRNA processing reactions and $r$-subunit assembly and maintain telomerase stability (reviewed in $[87,196]$ ). It has been proposed that all mature H/ACA snoRNPs are composed of a distinct box H/ACA snoRNA, which selectively base pairs with its target RNA, and two sets of four con- served core proteins, one per each hairpin motif of the bipartite structure of the snoRNA: the pseudouridinase Cbf5 (NAP57 in rodents and dyskerin in humans), Nop10, Nhp2 (L7Ae in archaea) and Gar1 [87, 196, 197]. Although these complexes can self-assemble in vitro (for a reference, see [198]), H/ACA snoRNP formation requires multiples trans-acting factors in vivo [197]. Among these factors, two conserved and essential proteins, Shq1 and Naf1, are specifically required for the stable accumulation of all box H/ACA snoRNAs, without being part of the mature particles in either the nucleolus or the Cajal bodies (e.g. [199-201], reviewed in [197]).

It has been shown that Shq1 functions in an early step of H/ACA snoRNP biogenesis [202, 203]. Shq1 apparently binds newly synthesized Cbf5 acting as a chaperone that prevents its misfolding, aggregation and degradation. Additionally, Shq1 prevents non-specific interactions of nascent Cbf5 with other RNAs before its association with box H/ACA snoRNAs [203]. The Shq1•Cbf5 interaction mainly occurs through the central and Shq1-specific C-terminal (SSD) domains of Shq1 and the RNA-binding interface of Cbf5 [203]. Indeed, it has been shown that Shq1 acts an RNA mimic; specific residues of Shq1 occupy the same position on nascent $\mathrm{Cbf} 5$ as selected RNA residues of an H/ACA snoRNA do within mature H/ACA snoRNPs [186, 187]. As a corollary, the binding of Cbf5 to either Shq1 or an H/ACA snoRNA is obligatorily mutually exclusive. The subsequent release of Shq1 by a specific chaperone complex, R2TP, allows the progression of H/ACA snoRNP biogenesis. Moreover, it has been proposed that another assembly factor, known as Naf1, brings Cbf5, Nop10 and Nhp2 to nascent box H/ACA RNAs at their site of transcription [196, 197].

Naf1 was identified as a nuclear factor able to interact with both the C-terminal domain of RNA polymerase II and nascent H/ACA snoRNAs [200]. At this time, a certain structural homology between Naf1 and the core domain of Gar1 was predicted [200], thus, being suggestive of a placeholder activity of Naf1 for Gar1. In agreement with this hypothesis, human NAF1 and GAR1 bind NAP57 (human Cbf5) competitively and in a mutually exclusive manner [204]. Moreover, crystallography demonstrated the structural homology between yeast Naf1 and the protein domain that in archaeal Gar1 binds Cbf5 [188]. Importantly, Naf1containing nascent H/ACA snoRNPs seem to be fully inactive regarding pseudouridinase activity; only the later replacement of Naf1 by Gar1 during H/ACA snoRNP biogenesis permits the formation of an active snoRNP [198, 204]. This latter result strongly suggests that the physiological function of Naf1, as the one of a placeholder factor, is keeping the emerging RNA-based machine inactive in order to prevent undesired effects on non-cognate substrates.

\section{FUTURE PERSPECTIVES AND CONCLUSIONS}

In this review, we have described a relatively large number of trans-acting assembly factors that work as placeholder factors during the ribosome biogenesis process. The placeholder hypothesis, which was initially suggested as the 
functional corollary of the presence of a few assembly factors showing a high degree of similarity to distinct $r$ proteins over their entire length [31], has been confirmed for more trans-acting factors than initially envisaged. This pleasant surprise has come as the experimental consequence of the combination of powerful biochemical, cell biological and genetic studies with the structural characterization of pre-ribosomal particles or reconstituted $r$ subunits by X-ray crystallography and cryo-EM from sub- to near-atomic resolution (e.g. [40]). This new mode to approach the pathway of ribosome biogenesis is providing for the first time clues on how trans-acting factors bind and operate to accommodate other factors or r-proteins during the maturation reactions of the nascent $r$-subunits (for a review, see [124]).

The phenotypic analyses of loss-of-function mutant variants of placeholder factors has proven to be very useful for revealing their functional relevance during the eukaryotic ribosome assembly process and have provided insights on why these factors have been positively selected during evolution. We have discussed several functions for placeholder factors: (i) Some placeholder factors (e.g. Nog2 or Mrt4) clearly control the position and timing of association or assembly of their counterparts. In the absence of these placeholder factors, the respective counterparts are prematurely recruited to the nascent pre-ribosomal particles, impeding important events that only the placeholder factors are able to exert. We can envisage a scenario where these placeholder factors are guarding key sites in premature $r$-particles from the binding of other factors or $r$ proteins. The premature recruitment of these laterassociating proteins could even generate kinetically trapped assembly intermediates that impede downstream maturation steps. However, in other cases (e.g. Rlp24), although the function of the placeholder factor has been shown essential for the correct progression of the maturation of the r-subunit, it is still unclear whether a direct functional relationship exists between the placeholder factor and its counterpart r-protein. (ii) Many of the strategies involving placeholder factors during ribosome biogenesis ensure that the nascent $r$-particles follow a stepdependent and ordered process of assembly. These steps are unidirectional and therefore irreversible. For example, nuclear pre-60S r-particles are unable to acquire export competence until Nog2 is replaced by Nmd3; cytoplasmic maturation of pre-60S r-particles does not properly proceed if Nog1 is not efficiently released and replaced by Rei1; Tsr1 delays association of either elF5B or Rio1 with cytoplasmic pre-40S r-particles. (iii) Specific dedicated chaperones and/or escortins carry out mutually exclusive interactions with other factors, rRNA or r-proteins. As discussed herein, these factors assist the import and assembly of distinct $r$-proteins while preventing their intrinsic tendency to aggregate, their degradation and/or their nonspecific association to non-cognate substrates. In some cases, these chaperones act as rRNA mimics when they exert their placeholding activity. By mimicking, chaperones and escortins are even able to guarantee the proper folding of their substrates. As also discussed, these factors are even able to promote the interdependent assembly of more than one r-protein at the same time ensuring stoichiometry. (iv) A considerable group of placeholder factors might have critical roles in structural proofreading, as previously discussed by A. W. Johnson [138]. A mechanism based on structural proofreading implies that the binding of a distinct factor or r-proteins depends on the proper generation of a specific site only after completion of selected upstream assembly steps. This strategy is used during export and cytoplasmic maturation of r-subunits, provides tools and mechanisms to detect assembly errors and ensures the specific cytoplasmic assembly of the last $r$ proteins. Equally important are strategies based on functional proofreading (discussed in [55, 122]) where key functional centres in r-subunits are inspected by a considerable number of factors, such as Arx 1 , which examines the solvent-exposed exit side of the PET, Nog1, Rei1, Reh1 and Sdo1, which probe the length and integrity of the PET, $\mathrm{Nmd3}$, which examines the E- and P-sites of nascent LSUs, Efl1, which together with Sdo1 inspects the integrity of the P-site and the GAC regions of nascent LSUs, the P-stalk protecting Mrt4, etc. This functional proofreading strategy ensures that only properly assembled nascent $r$-particles undergo final maturation while simultaneously preventing premature translation by masking the active sites of the ribosome.

There is plenty more work ahead to fully understand the exact function of most, if not all, placeholder factors and the exact relationship with their counterparts during ribosome assembly. Further work is required to acquire more data on the binding sites and complete 3D maps at high resolution of $r$-particles containing many of the placeholder factors so far known. These aspects are essential to obtain precise information on the location of the placeholder factors relative to the pre-rRNAs, other factors and $r$-proteins. For many of them, their structures are still unsolved (e.g. Drg1, Mex67, Nug2). This also applies to some of the dedicated chaperones and escortins so far identified (e.g. Bcp1, Rrb1, Tsr2). Most importantly, the exact sequence of the mechanistic events that lead to the exchange of a placeholder by its counterpart also remains to be dissected for many of the examples described herein.

Finally, we would like to remark that mutations in several placeholder factors have been linked to human diseases. (i) These include the Shwachman-Diamond Syndrome, where about $90 \%$ of the patients have mutations in the SBDS gene. These mutant variants have been shown to be defective in the release of Tif6 from cytoplasmic pre-60S rparticles (see, [116, 205], and reference therein). (ii) Moreover, mutations in the RPL10 gene have been identified in patients of T-cell acute lymphoblastic leukaemia and they also seem to impair the efficient release of Tif6 and $\mathrm{Nmd} 3$ from cytoplasmic pre-60S r-particles (see [206], and references therein). (iii) The Syndrome $5 q$ - seems to be caused by a RPS14 haploinsuficiency (reviewed in [207]), thus, it is reasonable to imagine that certain loss-of-function mutations in FAP7 could be identified in the future as linked to this disease, in a similar manner as mutations in RPS26 or its escortin TSR2 have been linked to the Diamond-Blackfan 
Anemia (discussed in [151]). (iv) Some mutations in dyskerin (human $\mathrm{Cbf5}$ ) related to $\mathrm{X}$-linked dyskeratosis congenita lead to the destabilization of the interaction of the mutant variant of dyskerin with its placeholder SHQ1 [187]. (v) Finally, mutually exclusive binding of MDM2 and $25 \mathrm{~S}$ rRNA to the 5S RNP is the basis of p53 activation and signalling in numerous pathophysiological situations (discussed in [191]). In conclusion, both the academic and the biomedical fields could benefit of the surely attractive and productive research on placeholder situations during ribosome biogenesis in the coming years.

\section{ACKNOWLEDGMENTS}

We apologize to all colleagues whose work could not be appropriately discussed in this review due to space constraints. We thank Dieter Kressler for helpful comments on the manuscript and Ana M. Rojas for help with the protein sequence alignments. This work has been supported by grants from the Spanish Ministry of Economy and Competitiveness (MINECO) and the ERDF (BFU2013-42958-P and BFU2016-75352-P) to

\section{REFERENCES}

1. Schmeing TM, Ramakrishnan V (2009). What recent ribosome structures have revealed about the mechanism of translation. Nature 461(7268): 1234-1242.

2. Warner JR (1999). The economics of ribosome biosynthesis in yeast. Trends Biochem Sci 24(11): 437-440.

3. Nomura $\mathrm{N}$ (1999). Regulation of ribosome biosynthesis in Escherichia coli and Saccharomyces cerevisiae: diversity and common principles. J Bacteriol 181(22): 6857-6864.

4. Wilson DN, Nierhaus KH (2007). The weird and wonderful world of bacterial ribosome regulation. Crit Rev Biochem Mol Biol 42(3): 187219

5. Hage AE, Tollervey D (2004). A surfeit of factors: why is ribosome assembly so much more complicated in eukaryotes than bacteria? RNA Biol 1(1): 10-15.

6. Shajani Z, Sykes MT, Williamson JR (2011). Assembly of bacterial ribosomes. Annu Rev Biochem 80: 501-526.

7. Omer AD, Zago M, Chang A, Dennis PP (2006). Probing the structure and function of an archaeal C/D-box methylation guide sRNA. RNA 12(9): 1708-1720.

8. Ebersberger I, Simm S, Leisegang MS, Schmitzberger P, Mirus O, von Haeseler A, Bohnsack MT, Schleiff E (2013). The evolution of the ribosome biogenesis pathway from a yeast perspective. Nucleic Acids Res 42(3): 1509-1523.

9. Piekna-Przybylska D, Decatur WA, Fournier MJ (2007). New bioinformatic tools for analysis of nucleotide modifications in eukaryotic rRNA. RNA 13(3): 305-312.

10. Woolford JL, Jr., Baserga SJ (2013). Ribosome biogenesis in the yeast Saccharomyces cerevisiae. Genetics 195(3): 643-681.

11. Tafforeau L, Zorbas $\mathrm{C}$, Langhendries JL, Mullineux ST, Stamatopoulou V, Mullier R, Wacheul L, Lafontaine DL (2013). The complexity of human ribosome biogenesis revealed by systematic nucleolar screening of Pre-rRNA processing factors. Mol Cell 51(4): 539-551.
JdIC. FJE-M and RB were supported by FPI fellowships from MINECO and the Andalusian Government, respectively.

\section{SUPPLEMENTAL MATERIAL}

All supplemental data for this article are available online at www.microbialcell.com.

\section{CONFLICT OF INTEREST}

The authors declare no conflict of interest.

\section{COPYRIGHT}

(C) 2017 Espinar-Marchena et al. This is an open-access article released under the terms of the Creative Commons Attribution (CC BY) license, which allows the unrestricted use, distribution, and reproduction in any medium, provided the original author and source are acknowledged.

Please cite this article as: Francisco J. Espinar-Marchena, Reyes Babiano and Jesús de la Cruz (2017). Placeholder factors in ribosome biogenesis: please, pave my way. Microbial Cell 4(5): 144168. doi: $10.15698 / \mathrm{mic} 2017.05 .572$

12. Lestrade L, Weber MJ (2006). snoRNA-LBME-db, a comprehensive database of human H/ACA and C/D box snoRNAs. Nucleic Acids Res 34(Database issue): D158-D162.

13. Kressler D, Hurt E, Bassler J (2010). Driving ribosome assembly. Biochim Biophys Acta 1803(6): 673-683.

14. Nerurkar P, Altvater M, Gerhardy S, Schütz S, Fischer U, Weirich C, Panse VG (2015). Eukaryotic Ribosome Assembly and Nuclear Export. Int Rev Cell Mol Biol 319: 107-140.

15. Iborra FJ, Jackson DA, Cook PR (2001). Coupled transcription and translation within nuclei of mammalian cells. Science 293(5532): 1193-1142.

16. Karbstein K (2013). Quality control mechanisms during ribosome maturation. Trends Cell Biol 23(5): 242-250.

17. de la Cruz J, Karbstein K, Woolford JL, Jr. (2015). Functions of ribosomal proteins in assembly of eukaryotic ribosomes in vivo. Annu Rev Biochem 84: 93-129.

18. Panse VG, Johnson AW (2010). Maturation of eukaryotic ribosomes: acquisition of functionality. Trends Biochem Sci 35(5): 260-266.

19. Senger B, Lafontaine DL, Graindorge JS, Gadal O, Camasses A, Sanni A, Garnier JM, Breitenbach M, Hurt E, Fasiolo F (2001). The nucle(ol)ar Tif6p and Efl1p are required for a late cytoplasmic step of ribosome synthesis. Mol Cell 8(6): 1363-1373.

20. Rodríguez-Mateos M, García-Gómez JJ, Francisco-Velilla R, Remacha M, de la Cruz J, Ballesta JPG (2009). Role and dynamics of the ribosomal protein $\mathrm{PO}$ and its related trans-acting factor Mrt4 during ribosome assembly in Saccharomyces cerevisiae. Nucleic Acids Res 37(22): 7519-7532.

21. Strunk BS, Loucks CR, Su M, Vashisth H, Cheng S, Schilling J, Brooks CL, III., Karbstein K, Skiniotis G (2011). Ribosome assembly factors prevent premature translation initiation by $40 \mathrm{~S}$ assembly intermediates. Science 333(6048): 1449-1453. 
22. Alves-Rodrigues I, Ferreira PG, Moldón A, Vivancos AP, Hidalgo E, Guigó R, Ayté J (2016). Spatiotemporal Control of Forkhead Binding to DNA Regulates the Meiotic Gene Expression Program. Cell Rep 14(4): 885-895.

23. Dunleavy EM, Almouzni G, Karpen GH (2011). H3.3 is deposited at centromeres in S phase as a placeholder for newly assembled CENP-A in $\mathrm{G}_{1}$ phase. Nucleus 2(2): 146-157.

24. Blum $R$ (2014). Activation of muscle enhancers by MyoD and epigenetic modifiers. J Cell Biochem 115(11): 1855-1867.

25. Warner JR (2001). Nascent ribosomes. Cell 107(2): 133-136.

26. Zuk D, Belk JP, Jacobson A (1999). Temperature-sensitive mutations in the Saccharomyces cerevisiae MRT4, GRC5, SLA2 and THS1 genes result in defects in mRNA turnover. Genetics 153(1): 3547.

27. Lalo D, Mariotte S, Thuriaux $P$ (1993). Two distinct yeast proteins are related to the mammalian ribosomal polypeptide L7. Yeast $9(10)$ : 1085-1091.

28. Saveanu C, Namane A, Gleizes PE, Lebreton A, Rousselle JC, Noaillac-Depeyre J, Gas N, Jacquier A, Fromont-Racine M (2003). Sequential protein association with nascent $60 \mathrm{~S}$ ribosomal particles. Mol Cell Biol 23(13): 4449-4460.

29. Rodríguez-Mateos M, Abia D, García-Gómez JJ, Morreale A, de la Cruz J, Santos C, Remacha M, Ballesta JPG (2009). The amino terminal domain from Mrt4 protein can functionally replace the RNA binding domain of the ribosomal P0 protein. Nucleic Acids Res 37(11): 35143521.

30. Harnpicharnchai $P$, Jakovljevic J, Horsey E, Miles T, Roman J, Rout M, Meagher D, Imai B, Guo Y, Brame CJ, Shabanowitz J, Hunt DF, Woolford JL, Jr. (2001). Composition and functional characterization of yeast 66S ribosome assembly intermediates. Mol Cell 8(3): 505-515.

31. Dunbar DA, Dragon F, Lee SJ, Baserga SJ (2000). A nucleolar protein related to ribosomal protein $\mathrm{L7}$ is required for an early step in large ribosomal subunit biogenesis. Proc Natl Acad Sci USA 97(24): 13027-13032.

32. Lee SJ, Baserga SJ (1999). Imp3p and Imp4p, two specific components of the U3 small nucleolar ribonucleoprotein that are essential for 18S rRNA processing. Mol Cell Biol 19(8): 5441-5452.

33. Ban N, Beckmann R, Cate JH, Dinman JD, Dragon $F$, Ellis SR, Lafontaine DL, Lindahl L, Liljas A, Lipton JM, McAlear MA, Moore PB, Noller HF, Ortega J, Panse VG, Ramakrishnan V, Spahn CM, Steitz TA, Tchórzewski M, Tollervey $D$, Warren AJ, Williamson JR, Wilson $D$, Yonath $A$, Yusupov $M$ (2014). A new system for naming ribosomal proteins. Curr Opin Struct Biol 24: 165-169.

34. Torchet C, Jacq C, Hermann-Le Denmat S (1998). Two mutant forms of the S1/TPR-containing protein Rrp5p affect the 18S rRNA synthesis in Saccharomyces cerevisiae. RNA 4(12): 1636-1652.

35. Mao H, White SA, Williamson JR (1999). A novel loop-loop recognition motif in the yeast ribosomal protein $L 30$ autoregulatory RNA complex. Nat Struct Biol 6(12): 1139-1147.

36. Santos C, Ballesta JPG (2005). Characterization of the 26S rRNAbinding domain in Saccharomyces cerevisiae ribosomal stalk phosphoprotein P0. Mol Microbiol 58(1): 217-226.

37. Santos C, Ballesta JPG (1995). The highly conserved protein PO carboxyl end is essential for ribosome activity only in the absence of proteins P1 and P2. J Biol Chem 270(35): 20608-20614.

38. Francisco-Velilla R, Remacha M, Ballesta JP (2013). Carboxy terminal modifications of the $\mathrm{PO}$ protein reveal alternative mechanisms of nuclear ribosomal stalk assembly. Nucleic Acids Res 41(18): 8628-8636.
39. Michalec B, Krokowski D, Grela P, Wawiorka L, Sawa-Makarska J, Grankowski N, Tchórzewski M (2010). Subcellular localization of ribosomal P0-like protein MRT4 is determined by its $\mathrm{N}$-terminal domain. Int J Biochem Cell Biol 42(5): 736-748.

40. Wu S, Tutuncuoglu B, Yan K, Brown H, Zhang Y, Tan D, Gamalinda M, Yuan Y, Li Z, Jakovljevic J, Ma C, Lei J, Dong MQ, Woolford JL, Jr., Gao N (2016). Diverse roles of assembly factors revealed by structures of late nuclear pre-60S ribosomes. Nature 534(7605): 133-137.

41. Leidig C, Thoms M, Holdermann I, Bradatsch B, Berninghausen O, Bange G, Sinning I, Hurt E, Beckmann R (2014). 60 S ribosome biogenesis requires rotation of the $5 S$ ribonucleoprotein particle. Nat Commun 5: 3491

42. Sarkar A, Pech M, Thoms M, Beckmann R, Hurt E (2016). Ribosome-stalk biogenesis is coupled with recruitment of nuclearexport factor to the nascent 605 subunit. Nat Struct Mol Biol 23(12): 1074-1082.

43. Ma C, Wu S, Li N, Chen Y, Yan K, Li Z, Zheng L, Lei J, Woolford JL, Jr., Gao N (2017). Structural snapshot of cytoplasmic pre-60S ribosomal particles bound by Nmd3, Lsg1, Tif6 and Reh1. Nat Struct Mol Biol 24(3): 214-220.

44. Ben-Shem A, Garreau de Loubresse N, Melnikov S, Jenner L, Yusupova G, Yusupov M (2011). The structure of the eukaryotic ribosome at 3.0 Å resolution. Science 334(6062): 1524-1529.

45. Kemmler S, Occhipinti L, Veisu M, Panse VG (2009). Yvh1 is required for a late maturation step in the 605 biogenesis pathway. J Cell Biol 186(6): 863-880.

46. Lo KY, Li Z, Wang F, Marcotte EM, Johnson AW (2009). Ribosome stalk assembly requires the dual-specificity phosphatase Yvh1 for the exchange of Mrt4 with P0. J Cell Biol 186(6): 849-862.

47. Klinge S, Voigts-Hoffmann F, Leibundgut M, Arpagaus S, Ban N (2011). Crystal structure of the eukaryotic 60S ribosomal subunit in complex with initiation factor 6. Science 334(6058): 941-948.

48. Wawiórka L, Molestak E, Szajwaj M, Michalec-Wawiórka B, Boguszewska A, Borkiewicz L, Liudkovska V, Kufel J, Tchórzewski M (2016). Functional analysis of the uL11 protein impact on translational machinery. Cell Cycle 15(8): 1060-1072.

49. Yao W, Lutzmann M, Hurt E (2008). A versatile interaction platform on the Mex67-Mtr2 receptor creates an overlap between mRNA and ribosome export. EMBO J 27(1): 6-16.

50. Yao W, Roser D, Kohler A, Bradatsch B, Bassler J, Hurt E (2007). Nuclear export of ribosomal 60S subunits by the general mRNA export receptor Mex67-Mtr2. Mol Cell 26(1): 51-62.

51. Thoms M, Thomson E, Bassler J, Gnadig M, Griesel S, Hurt E (2015). The Exosome Is Recruited to RNA Substrates through Specific Adaptor Proteins. Cell 162(5): 1029-1038.

52. Fernández-Pevida A, Kressler D, de la Cruz J (2015). Processing of preribosomal RNA in Saccharomyces cerevisiae. Wiley Interdiscip Rev RNA 6(2): 191-209.

53. Talkish J, Zhang J, Jakovljevic J, Horsey EW, Woolford JL, Jr. (2012). Hierarchical recruitment into nascent ribosomes of assembly factors required for 27SB pre-rRNA processing in Saccharomyces cerevisiae. Nucleic Acids Res 40(17): 8646-8661.

54. Saveanu C, Rousselle JC, Lenormand P, Namane A, Jacquier A, Fromont-Racine $M$ (2007). The p21-activated protein kinase inhibitor Skb15 and its budding yeast homologue are 60S ribosome assembly factors. Mol Cell Biol 27(8): 2897-2909.

55. Lo KY, Li Z, Bussiere C, Bresson S, Marcotte EM, Johnson AW (2010). Defining the pathway of cytoplasmic maturation of the 60S ribosomal subunit. Mol Cell 39(2): 196-208. 
56. Pertschy B, Saveanu C, Zisser G, Lebreton A, Tengg M, Jacquier A, Liebminger E, Nobis B, Kappel L, van der Klei I, Hogenauer G, FromontRacine $\mathrm{M}$, Bergler $\mathrm{H}$ (2007). Cytoplasmic recycling of 60S preribosomal factors depends on the AAA protein Drg1. Mol Cell Biol 27(19): 65816592.

57. Bassler J, Klein I, Schmidt C, Kallas M, Thomson E, Wagner MA, Bradatsch B, Rechberger G, Strohmaier H, Hurt E, Bergler H (2012). The conserved Bud20 zinc finger protein is a new component of the ribosomal 60S subunit export machinery. Mol Cell Biol 32(24): 48984912.

58. Altvater $M$, Chang $Y$, Melnik $A$, Occhipinti L, Schütz $S$, Rothenbusch U, Picotti P, Panse VG (2012). Targeted proteomics reveals compositional dynamics of 605 pre-ribosomes after nuclear export. Mol Syst Biol 8: 628.

59. Kressler D, Hurt E, Bergler H, Bassler J (2012). The power of AAAATPases on the road of pre-60S ribosome maturation--molecular machines that strip pre-ribosomal particles. Biochim Biophys Acta 1823(1): 92-100.

60. Kappel L, Loibl M, Zisser G, Klein I, Fruhmann G, Gruber C, Unterweger S, Rechberger G, Pertschy B, Bergler H (2012). Rlp24 activates the AAA-ATPase Drg1 to initiate cytoplasmic pre-60S maturation. J Cell Biol 199(5): 771-782.

61. Loibl M, Klein I, Prattes M, Schmidt C, Kappel L, Zisser G, Gungl A, Krieger E, Pertschy B, Bergler H (2014). The drug diazaborine blocks ribosome biogenesis by inhibiting the AAA-ATPase Drg1. J Biol Chem 289(7): 3913-3922.

62. Lebreton A, Saveanu C, Decourty L, Rain JC, Jacquier A, FromontRacine $M$ (2006). A functional network involved in the recycling of nucleocytoplasmic pre-60S factors. J Cell Biol 173(3): 349-360.

63. Parnell KM, Bass BL (2009). Functional redundancy of yeast proteins Reh1 and Rei1 in cytoplasmic 60S subunit maturation. Mol Cell Biol 29(14): 4014-4023.

64. Demoinet $E$, Jacquier A, Lutfalla G, Fromont-Racine M (2007). The $\mathrm{Hsp} 40$ chaperone Jjj1 is required for the nucleo-cytoplasmic recycling of preribosomal factors in Saccharomyces cerevisiae. RNA 13(9): 15701581.

65. Meyer AE, Hung NJ, Yang P, Johnson AW, Craig EA (2007). The specialized cytosolic J-protein, Jjj1, functions in $60 \mathrm{~S}$ ribosomal subunit biogenesis. Proc Natl Acad Sci USA 104(5): 1558-1563.

66. Meyer AE, Hoover LA, Craig EA (2010). The cytosolic J-protein, Jjj1, and Rei1 function in the removal of the pre-60 S subunit factor Arx 1 . J Biol Chem 285(2): 961-968.

67. Hung NJ, Johnson AW (2006). Nuclear recycling of the pre-60S ribosomal subunit-associated factor Arx1 depends on Rei1 in Saccharomyces cerevisiae. Mol Cell Biol 26(10): 3718-3727.

68. Greber BJ, Gerhardy S, Leitner A, Leibundgut $M$, Salem $M$, Boehringer D, Leulliot N, Aebersold R, Panse VG, Ban N (2016). Insertion of the biogenesis factor Rei1 probes the ribosomal tunnel during 605 maturation. Cell 164(1-2): 91-102.

69. Greber BJ, Boehringer D, Montellese C, Ban N (2012). Cryo-EM structures of Arx1 and maturation factors Rei1 and Jjj1 bound to the 60 S ribosomal subunit. Nat Struct Mol Biol 19(12): 1228-1233.

70. Bradatsch B, Leidig C, Granneman S, Gnadig M, Tollervey D, Bottcher B, Beckmann R, Hurt E (2012). Structure of the pre-60S ribosomal subunit with nuclear export factor Arx1 bound at the exit tunnel. Nat Struct Mol Biol 19(12): 1234-1241.

71. Babiano R, Badis G, Saveanu C, Namane A, Doyen A, Diaz-Quintana $A$, Jacquier A, Fromont-Racine $M$, de la Cruz J (2013). Yeast ribosomal protein L7 and its homologue RIp7 are simultaneously present at distinct sites on pre-60S ribosomal particles. Nucleic Acids Res 41(20): 9461-9470.

72. Dembowski JA, Ramesh M, McManus CJ, Woolford JL, Jr. (2013). Identification of the binding site of Rlp7 on assembling 60S ribosomal subunits in Saccharomyces cerevisiae. RNA 19(12): 1639-1647.

73. Sahasranaman A, Dembowski J, Strahler J, Andrews P, Maddock J, Woolford JL, Jr. (2011). Assembly of Saccharomyces cerevisiae $60 \mathrm{~S}$ ribosomal subunits: role of factors required for $27 \mathrm{~S}$ pre-rRNA processing. EMBO J 30(19): 4020-4032.

74. Gadal O, Strauss D, Petfalski E, Gleizes PE, Gas N, Tollervey D, Hurt E (2002). Rlp7p is associated with 605 preribosomes, restricted to the granular component of the nucleolus, and required for pre-rRNA processing. J Cell Biol 157(6): 941-951.

75. Granneman S, Petfalski E, Tollervey D (2011). A cluster of ribosome synthesis factors regulate pre-rRNA folding and 5.8S rRNA maturation by the Rat1 exonuclease. EMBO J 30(19): 4006-4019.

76. Tutuncuoglu B, Jakovljevic J, Wu S, Gao N, Woolford JL, Jr. (2016). The $\mathrm{N}$-terminal extension of yeast ribosomal protein $\mathrm{L} 8$ is involved in two major remodeling events during late nuclear stages of $60 \mathrm{~S}$ ribosomal subunit assembly. RNA 22(9): 1386-1399.

77. Jakovljevic J, Ohmayer U, Gamalinda M, Talkish J, Alexander L, Linnemann J, Milkereit P, Woolford JL, Jr. (2012). Ribosomal proteins L7 and L8 function in concert with six $A_{3}$ assembly factors to propagate assembly of domains I and II of 25S rRNA in yeast 60S ribosomal subunits. RNA 18(10): 1805-1822.

78. Pöll G, Braun T, Jakovljevic J, Neueder A, Jakob S, Woolford JL, Jr., Tschochner $\mathrm{H}$, Milkereit $\mathrm{P}$ (2009). rRNA maturation in yeast cells depleted of large ribosomal subunit proteins. PLoS One 4(12): e8249.

79. Ohmayer $U$, Gil-Hernández A, Sauert $M$, Martín-Marcos $P$, Tamame M, Tschochner H, Griesenbeck J, Milkereit P (2015). Studies on the coordination of ribosomal protein assembly events involved in processing and stabilization of yeast early large ribosomal subunit precursors. PLoS One 10(12): e0143768.

80. Konikkat S, Woolford JL, Jr. (2016). Principles of $60 \mathrm{~S}$ ribosomal subunit assembly emerging from recent studies in yeast. Biochem J 474(2): 195-214.

81. Wehner KA, Gallagher JE, Baserga SJ (2002). Components of an interdependent unit within the SSU processome regulate and mediate its activity. Mol Cell Biol 22(20): 7258-7267.

82. Pérez-Fernández J, Román A, de las Rivas J, Bustelo XR, Dosil M (2007). The $90 \mathrm{~S}$ preribosome is a multimodular structure that is assembled through a hierarchical mechanism. Mol Cell Biol 27(15): 5414-5429.

83. Pérez-Fernández J, Martín-Marcos P, Dosil M (2011). Elucidation of the assembly events required for the recruitment of Utp20, Imp4 and Bms1 onto nascent pre-ribosomes. Nucleic Acids Res 39(18): 81058121.

84. Chaker-Margot M, Hunziker M, Barandun J, Dill BD, Klinge S (2015). Stage-specific assembly events of the 6-MDa small-subunit processome initiate eukaryotic ribosome biogenesis. Nat Struct Mol Biol 22(11): 920-923.

85. Gérczei T, Correll CC (2004). Imp3p and Imp4p mediate formation of essential U3-precursor rRNA (pre-rRNA) duplexes, possibly to recruit the small subunit processome to the pre-rRNA. Proc Natl Acad Sci USA 101(43): 15301-15306.

86. Shah BN, Liu X, Correll CC (2013). Imp3 unfolds stem structures in pre-rRNA and U3 snoRNA to form a duplex essential for small subunit processing. RNA 19(10): 1372-1383. 
87. Watkins NJ, Bohnsack MT (2012). The box C/D and H/ACA snoRNPs: key players in the modification, processing and the dynamic folding of ribosomal RNA. Wiley Interdiscip Rev RNA 3(3): 397-414.

88. Ferreira-Cerca S, Pöll G, Gleizes PE, Tschochner H, Milkereit $P$ (2005). Roles of eukaryotic ribosomal proteins in maturation and transport of pre-18S rRNA and ribosome function. Mol Cell 20(2): 263275.

89. Ferreira-Cerca S, Pöll G, Kuhn H, Neueder A, Jakob S, Tschochner H, Milkereit $P$ (2007). Analysis of the in vivo assembly pathway of eukaryotic 40S ribosomal proteins. Mol Cell 28(3): 446-457.

90. O'Donohue MF, Choesmel V, Faubladier M, Fichant G, Gleizes PE (2010). Functional dichotomy of ribosomal proteins during the synthesis of mammalian 40 S ribosomal subunits. J Cell Biol 190(5): 853-866.

91. Rabl J, Leibundgut M, Ataide SF, Haag A, Ban N (2011). Crystal structure of the eukaryotic $40 \mathrm{~S}$ ribosomal subunit in complex with initiation factor 1. Science 331(6018): 730-736.

92. Amlacher S, Sarges P, Flemming D, van Noort V, Kunze R, Devos DP, Arumugam $M$, Bork $P$, Hurt $E$ (2011). Insight into structure and assembly of the nuclear pore complex by utilizing the genome of a eukaryotic thermophile. Cell 146(2): 277-289.

93. Chaker-Margot M, Barandun J, Hunziker M, Klinge S (2016). Architecture of the yeast small subunit processome. Science 355(6321).

94. Kornprobst $M$, Turk M, Kellner N, Cheng J, Flemming D, Kos-Braun I, Kos M, Thoms M, Berninghausen O, Beckmann R, Hurt E (2016). Architecture of the $90 \mathrm{~S}$ pre-ribosome: a structural view on the birth of the eukaryotic ribosome. Cell 166(2): 380-393.

95. Sun Q, Zhu X, Qi J, An W, Lan P, Tan D, Chen R, Wang B, Zheng S, Zhang C, Chen X, Zhang W, Chen J, Dong MQ, Ye K (2017). Molecular architecture of the $90 \mathrm{~S}$ small subunit pre-ribosome. elife 6.

96. Grandi P, Rybin V, Bassler J, Petfalski E, Strauss D, Marzioch M, Schäfer T, Kuster B, Tschochner H, Tollervey D, Gavin AC, Hurt E (2002). $90 \mathrm{~S}$ pre-ribosomes include the $35 \mathrm{~S}$ pre-rRNA, the U3 snoRNP, and $40 \mathrm{~S}$ subunit processing factors but predominantly lack $60 \mathrm{~S}$ synthesis factors. Mol Cell 10(1): 105-115.

97. Dragon F, Gallagher JE, Compagnone-Post PA, Mitchell BM, Porwancher KA, Wehner KA, Wormsley S, Settlage RE, Shabanowitz J, Osheim Y, Beyer AL, Hunt DF, Baserga SJ (2002). A large nucleolar U3 ribonucleoprotein required for $18 \mathrm{~S}$ ribosomal RNA biogenesis. Nature 417(6892): 967-970.

98. Bernstein KA, Gallagher JE, Mitchell BM, Granneman S, Baserga SJ (2004). The small-subunit processome is a ribosome assembly intermediate. Eukaryot Cell 3(6): 1619-1626.

99. Gelperin D, Horton L, Beckman J, Hensold J, Lemmon SK (2001). Bms1p, a novel GTP-binding protein, and the related Tsr1p are required for distinct steps of $40 \mathrm{~S}$ ribosome biogenesis in yeast. RNA 7(9): 1268-1283.

100. Schäfer T, Strauss D, Petfalski E, Tollervey D, Hurt E (2003). The path from nucleolar 905 to cytoplasmic $40 S$ pre-ribosomes. EMBO J 22(6): 1370-1380.

101. Schäfer T, Maco B, Petfalski E, Tollervey D, Bottcher B, Aebi U, Hurt E (2006). Hrr25-dependent phosphorylation state regulates organization of the pre-40S subunit. Nature 441(7093): 651-655.

102. McCaughan UM, Jayachandran U, Shchepachev V, Chen ZA, Rappsilber J, Tollervey D, Cook AG (2016). Pre-40S ribosome biogenesis factor Tsr1 is an inactive structural mimic of translational GTPases. Nat Commun 7: 11789.
103. Granneman S, Petfalski E, Swiatkowska A, Tollervey D (2010). Cracking pre-40S ribosomal subunit structure by systematic analyses of RNA-protein cross-linking. EMBO J 29(12): 2026-2036.

104. Strunk BS, Novak MN, Young CL, Karbstein K (2012). A translation-like cycle is a quality control checkpoint for maturing $40 \mathrm{~S}$ ribosome subunits. Cell 150(1): 111-121.

105. Wegierski T, Billy E, Nasr F, Filipowicz W (2001). Bms1p, a Gdomain-containing protein, associates with Rcl1p and is required for 18S rRNA biogenesis in yeast. RNA 7(9): 1254-1267.

106. Zhang L, Wu C, Cai G, Chen S, Ye K (2016). Stepwise and dynamic assembly of the earliest precursors of small ribosomal subunits in yeast. Genes Dev 30(6): 718-732.

107. Ho JH-N, Kallstrom G, Johnson AW (2000). Nmd3p is a Crm1pdependent adapter protein for nuclear export of the large ribosomal subunit. J Cell Biol 151(5): 1057-1066.

108. Gadal O, Strauss D, Kessl J, Trumpower B, Tollervey D, Hurt E (2001). Nuclear export of 60S ribosomal subunit depends on Xpo1p and requires a nuclear export sequence-containing factor, Nmd3p, that associates with the large subunit protein Rpl10p. Mol Cell Biol 21(10): 3405-3415.

109. Ho JH-N, Johnson AW (1999). NMD3 encodes an essential cytoplasmic protein required for stable 60S ribosomal subunits in Saccharomyces cerevisiae. Mol Cell Biol 19(3): 2389-2399.

110. West M, Hedges JB, Chen A, Johnson AW (2005). Defining the order in which Nmd3p and Rpl10p load onto nascent 60S ribosomal subunits. Mol Cell Biol 25(9): 3802-3813.

111. Hedges J, West M, Johnson AW (2005). Release of the export adapter, Nmd3p, from the 60S ribosomal subunit requires Rpl10p and the cytoplasmic GTPase Lsg1p. EMBO J 24(3): 567-579.

112. Pachler K, Karl T, Kolmann K, Mehlmer N, Eder M, Loeffler M, Oender K, Hochleitner EO, Lottspeich F, Bresgen N, Richter K, Breitenbach $M$, Koller $L$ (2004). Functional interaction in establishment of ribosomal integrity between small subunit protein rpS6 and translational regulator rpL10/Grc5p. FEMS Yeast Res 5(3): 271-280.

113. Sengupta J, Bussiere C, Pallesen J, West M, Johnson AW, Frank J (2010). Characterization of the nuclear export adaptor protein Nmd3 in association with the 60S ribosomal subunit. J Cell Biol 189(7): 10791086.

114. Malyutin AG, Musalgaonkar S, Patchett S, Frank J, Johnson AW (2017). Nmd3 is a structural mimic of elF5A, and activates the cpGTPase Lsg1 during 60S ribosome biogenesis. EMBO J 36(7): 854868.

115. Matsuo Y, Granneman S, Thoms M, Manikas RG, Tollervey D, Hurt E (2014). Coupled GTPase and remodelling ATPase activities form a checkpoint for ribosome export. Nature 505(7481): 112-116.

116. Weis F, Giudice E, Churcher M, Jin L, Hilcenko C, Wong CC, Traynor D, Kay RR, Warren AJ (2015). Mechanism of elF6 release from the nascent 605 ribosomal subunit. Nat Struct Mol Biol 22(11): 914919.

117. Fernández-Pevida A, Rodríguez-Galán O, Díaz-Quintana A, Kressler D, de la Cruz J (2012). Yeast ribosomal protein $L 40$ assembles late into precursor 60S ribosomes and is required for their cytoplasmic maturation. J Biol Chem 287(45): 38390-38407.

118. Manikas RG, Thomson E, Thoms M, Hurt E (2016). The K+dependent GTPase Nug1 is implicated in the association of the helicase Dbp10 to the immature peptidyl transferase centre during ribosome maturation. Nucleic Acids Res 44(4): 1800-1812.

119. Bécam AM, Nasr F, Racki WJ, Zagulski M, Herbert CJ (2001). Ria1p (YnI163c), a protein similar to elongation factors 2, is involved in the 
biogenesis of the $60 \mathrm{~S}$ subunit of the ribosome in Saccharomyces cerevisiae. Mol Genet Genomics 266(3): 454-462.

120. Menne TF, Goyenechea B, Sanchez-Puig N, Wong CC, Tonkin LM, Ancliff PJ, Brost RL, Costanzo M, Boone C, Warren AJ (2007). The Shwachman-Bodian-Diamond syndrome protein mediates translational activation of ribosomes in yeast. Nat Genet 39(4): 486495.

121. Finch AJ, Hilcenko C, Basse N, Drynan LF, Goyenechea B, Menne TF, Gonzalez Fernandez A, Simpson P, D'Santos CS, Arends MJ Donadieu J, Bellanne-Chantelot C, Costanzo M, Boone C, McKenzie AN Freund SM, Warren AJ (2011). Uncoupling of GTP hydrolysis from elF6 release on the ribosome causes Shwachman-Diamond syndrome. Genes Dev 25(9): 917-929.

122. Bussiere C, Hashem Y, Arora S, Frank J, Johnson AW (2012). Integrity of the P-site is probed during maturation of the $60 \mathrm{~S}$ ribosomal subunit. J Cell Biol 197(6): 747-759.

123. Gartmann M, Blau M, Armache JP, Mielke T, Topf M, Beckmann R (2010). Mechanism of elF6-mediated inhibition of ribosomal subunit joining. J Biol Chem 285(20): 14848-14851.

124. Greber BJ (2016). Mechanistic insight into eukaryotic 60S ribosomal subunit biogenesis by cryo-electron microscopy. RNA 22(11): 1643-1662.

125. Baxter-Roshek JL, Petrov AN, Dinman JD (2007). Optimization of ribosome structure and function by rRNA base modification. PloS One 2(1): e174.

126. Bonnerot C, Pintard L, Lutfalla G (2003). Functional redundancy of Spb1p and a snR52-dependent mechanism for the 2'-O-ribose methylation of a conserved rRNA position in yeast. Mol Cell 12(5): 1309-1315.

127. Sharma S, Yang J, Watzinger $P$, Kötter $P$, Entian KD (2013). Yeat Nop2 and Rcm1 methylate C2870 and C2278 of the 25S rRNA, respectively. Nucleic Acids Res 41(19): 9062-9076.

128. Maden BE, Hughes JM (1997). Eukaryotic ribosomal RNA: the recent excitement in the nucleotide modification problem. Chromosoma 105(7-8): 391-400.

129. Lebreton A, Saveanu C, Decourty L, Jacquier A, Fromont-Racine $M$ (2006). Nsa2 is an unstable, conserved factor required for the maturation of 27SB pre-rRNAs. J Biol Chem 281(37): 27099-27108.

130. Bassler J, Paternoga H, Holdermann I, Thoms M, Granneman S, Barrio-Garcia C, Nyarko A, Lee W, Stier G, Clark SA, Schraivogel D, Kallas M, Beckmann R, Tollervey D, Barbar E, Sinning I, Hurt E (2015). A network of assembly factors is involved in remodeling rRNA elements during preribosome maturation. J Cell Biol 207(4): 481-498.

131. Lebreton A, Rousselle JC, Lenormand P, Namane A, Jacquier A, Fromont-Racine $M$, Saveanu $C$ (2008). 60S ribosomal subunit assembly dynamics defined by semi-quantitative mass spectrometry of purified complexes. Nucleic Acids Res 36(15): 4988-4999.

132. Bassler J, Kallas M, Hurt E (2006). The NUG1 GTPase reveals and $\mathrm{N}$-terminal RNA-binding domain that is essential for association with 60 S pre-ribosomal particles. J Biol Chem 281(34): 24737-24744.

133. Bradatsch B, Katahira J, Kowalinski E, Bange G, Yao W, Sekimoto T, Baumgartel V, Boese G, Bassler J, Wild K, Peters R, Yoneda Y, Sinning I, Hurt E (2007). Arx1 functions as an unorthodox nuclear export receptor for the 60S preribosomal subunit. Mol Cell 27(5): 767-779.

134. Hung NJ, Lo KY, Patel SS, Helmke K, Johnson AW (2008). Arx1 is a nuclear export receptor for the $60 \mathrm{~S}$ ribosomal subunit in yeast. Mol Biol Cell 19(2): 735-744.

135. Yao $Y$, Demoinet $E$, Saveanu C, Lenormand $P$, Jacquier $A$, Fromont-Racine $M$ (2010). Ecm1 is a new pre-ribosomal factor involved in pre-60S particle export. RNA 16(5): 1007-1017.
136. Nyathi Y, Pool MR (2015). Analysis of the interplay of protein biogenesis factors at the ribosome exit site reveals new role for NAC. J Cell Biol 210(2): 287-301.

137. Babiano R, de la Cruz J (2010). Ribosomal protein L35 is required for 27SB pre-rRNA processing in Saccharomyces cerevisiae Nucleic Acids Res 38(15): 5177-5192.

138. Johnson AW, Lund E, Dahlberg JE (2002). Nuclear export of ribosomal subunits. Trends Biochem Sci 27(11): 580-585.

139. Kramer G, Boehringer D, Ban N, Bukau B (2009). The ribosome as a platform for co-translational processing, folding and targeting of newly synthesized proteins. Nat Struct Mol Biol 16(6): 589-597.

140. Gerhardy S, Menet AM, Pena C, Petkowski JJ, Panse VG (2014). Assembly and nuclear export of pre-ribosomal particles in budding yeast. Chromosoma 123(4): 327-344.

141. Melnikov S, Ben-Shem A, Yusupova G, Yusupov M (2015). Insights into the origin of the nuclear localization signals in conserved ribosomal proteins. Nat Commun 6: 7382.

142. Rout MP, Blobel G, Aitchison JD (1997). A distinct nuclear import pathway used by ribosomal proteins. Cell 89(5): 715-725.

143. Schütz $S$, Fischer $U$, Altvater $M$, Nerurkar $P$, Pena $C$, Gerber $M$, Chang Y, Caesar S, Schubert OT, Schlenstedt G, Panse VG (2014). A RanGTP-independent mechanism allows ribosomal protein nuclear import for ribosome assembly. eLife 3: e03473.

144. Kressler D, Bange G, Ogawa Y, Stjepanovic G, Bradatsch B, Pratte D, Amlacher S, Strauss D, Yoneda Y, Katahira J, Sinning I, Hurt E (2012). Synchronizing nuclear import of ribosomal proteins with ribosome assembly. Science 338(6107): 666-671.

145. Bange G, Murat G, Sinning I, Hurt E, Kressler D (2013). New twist to nuclear import: When two travel together. Commun Integr Biol 6(4): e24792.

146. Jäkel S, Mingot JM, Schwarzmaier P, Hartmann E, Görlich D (2002). Importins fulfil a dual function as nuclear import receptors and cytoplasmic chaperones for exposed basic domains. EMBO J 21(3): 377-386.

147. Sung MK, Porras-Yakushi TR, Reitsma JM, Huber FM, Sweredoski MJ, Hoelz A, Hess S, Deshaies RJ (2016). A conserved quality-control pathway that mediates degradation of unassembled ribosomal proteins. elife 5: e19105.

148. Sydorskyy Y, Dilworth DJ, Yi EC, Goodlett DR, Wozniak RW, Aitchison JD (2003). Intersection of the Kap123p-mediated nuclear import and ribosome export pathways. Mol Cell Biol 23(6): 2042-2054.

149. Huber FM, Hoelz A (2017). Molecular basis for protection of ribosomal protein $\mathrm{L} 4$ from cellular degradation. Nat Commun 8: 14354.

150. Mitterer V, Gantenbein N, Birner-Gruenberger R, Murat G, Bergler H, Kressler D, Pertschy B (2016). Nuclear import of dimerized ribosomal protein Rps3 in complex with its chaperone Yar1. Sci Rep 6: 36714.

151. Pillet B, Mitterer V, Kressler D, Pertschy B (2016). Hold on to your friends: Dedicated chaperones of ribosomal proteins: Dedicated chaperones mediate the safe transfer of ribosomal proteins to their site of pre-ribosome incorporation. Bioessays 39(1): 1-12.

152. Pausch $P$, Singh $U$, Ahmed $Y L$, Pillet B, Murat G, Altegoer $F$, Stier G, Thoms M, Hurt E, Sinning I, Bange G, Kressler D (2015). Cotranslational capturing of nascent ribosomal proteins by their dedicated chaperones. Nat Commun 6: 7494.

153. Stelter $P$, Huber FM, Kunze R, Flemming D, Hoelz A, Hurt E (2015). Coordinated Ribosomal L4 Protein Assembly into the Pre-Ribosome Is Regulated by Its Eukaryote-Specific Extension. Mol Cell 58(5): 854-862. 
154. Pillet B, García-Gómez JJ, Pausch P, Falquet L, Bange G, de la Cruz J, Kressler D (2015). The dedicated chaperone Acl4 escorts ribosomal protein Rpl4 to its nuclear pre-60S assembly site. PLoS Genet 11(10): e1005565.

155. Zhang $Y$, Wolfle $T$, Rospert S (2013). Interaction of nascent chains with the ribosomal tunnel proteins Rpl4, Rpl17, and Rpl39 of Saccharomyces cerevisiae. J Biol Chem 288(47): 33697-33707.

156. Gamalinda M, Woolford JL, Jr. (2014). Deletion of L4 domains reveals insights into the importance of ribosomal protein extensions in eukaryotic ribosome assembly. RNA 20(11): 1725-1731.

157. louk TL, Aitchison JD, Maguire S, Wozniak RW (2001). Rrb1p, a yeast nuclear WD-repeat protein involved in the regulation of ribosome synthesis. Mol Cell Biol 21(4): 1260-1271.

158. Schaper S, Fromont-Racine M, Linder P, de la Cruz J, Namade A, Yaniv $M$ (2001). A yeast homolog of chromatin assembly factor 1 is involved in early ribosome assembly. Curr Biol 11(23): 1885-1890.

159. Meskauskas A, Dinman JD (2008). Ribosomal protein L3 functions as a 'rocker switch' to aid in coordinating of large subunit-associated functions in eukaryotes and Archaea. Nucleic Acids Res 36(19): 61756186.

160. Rosado IV, Dez C, Lebaron S, Caizergues-Ferrer M, Henry Y, de la Cruz J (2007). Characterization of Saccharomyces cerevisiae Npa2p (Urb2p) reveals a low-molecular-mass complex containing Dbp6p, Npa1p (Urb1p), Nop8p, and Rsa3p involved in early steps of 605 ribosomal subunit biogenesis. Mol Cell Biol 27(4): 1207-1221.

161. Eisinger DP, Dick FA, Denke E, Trumpower BL (1997). SQT1, which encodes an essential WD domain protein of Saccharomyces cerevisiae, suppresses dominant-negative mutations of the ribosomal protein gene QSR1. Mol Cell Biol 17(9): 5146-5155.

162. Frénois $F$, Legrand $P$, Fribourg $S$ (2016). Sqt1p is an eight-bladed WD40 protein. Acta Crystallogr F Struct Biol Commun 72(Pt 1): 59-64.

163. Calviño FR, Kharde S, Ori A, Hendricks A, Wild K, Kressler D, Bange G, Hurt E, Beck M, Sinning I (2015). Symportin 1 chaperones $5 S$ RNP assembly during ribosome biogenesis by occupying an essential rRNA-binding site. Nat Commun 6: 6510.

164. Zheng J, Lang $Y$, Zhang $Q$, Cui D, Sun H, Jiang L, Chen Z, Zhang R, Gao Y, Tian W, Wu W, Tang J (2015). Structure of human MDM2 complexed with RPL11 reveals the molecular basis of p53 activation. Genes Dev 29(14): 1524-1534.

165. Dechampesme A-M, Koroleva O, Léger-Silvestre I, Gas N, Camier $S$ (1999). Assembly of $5 S$ ribosomal RNA is required at a specific step of the pre-rRNA processing pathway. J Cell Biol 145(7): 1369-1380.

166. Zhang J, Harnpicharnchai P, Jakovljevic J, Tang L, Guo Y, Oeffinger M, Rout MP, Hiley SL, Hughes T, Woolford JL, Jr. (2007). Assembly factors Rpf2 and Rrs1 recruit 5S rRNA and ribosomal proteins rpL5 and rpL11 into nascent ribosomes. Genes Dev 21(20): 2580-2592.

167. Kharde S, Calviño FR, Gumiero A, Wild K, Sinning I (2015). The structure of Rpf2-Rrs1 explains its role in ribosome biogenesis. Nucleic Acids Res 43(14): 7083-7095.

168. Asano N, Kato K, Nakamura A, Komoda K, Tanaka I, Yao M (2015). Structural and functional analysis of the Rpf2-Rrs1 complex in ribosome biogenesis. Nucleic Acids Res 43(9): 4746-4457.

169. Madru C, Lebaron S, Blaud M, Delbos L, Pipoli J, Pasmant E, Réty S, Leulliot N (2015). Chaperoning 5S RNA assembly. Genes Dev 29(13): 1432-1446.

170. Peng WT, Robinson MD, Mnaimneh S, Krogan NJ, Cagney G, Morris Q, Davierwala AP, Grigull J, Yang X, Zhang W, Mitsakakis N, Ryan OW, Datta N, Jojic V, Pal C, Canadien V, Richards D, Beattie B, Wu LF, Altschuler SJ, Roweis S, Frey BJ, Emili A, Greenblatt JF, Hughes
TR (2003). A panoramic view of yeast noncoding RNA processing. Cell 113(7): 919-933.

171. Peña C, Schütz S, Fischer U, Chang Y, Panse VG (2016). Prefabrication of a ribosomal protein subcomplex essential for eukaryotic ribosome formation. elife 5: e21755.

172. Li Z, Lee I, Moradi E, Hung NJ, Johnson AW, Marcotte EM (2009). Rational extension of the ribosome biogenesis pathway using network-guided genetics. PLoS Biol 7(10): e1000213.

173. Ting YH, Lu TJ, Johnson AW, Shie JT, Chen BR, Kumar SS, Lo KY (2017). Bcp1 is the Nuclear Chaperone of the 60S ribosomal protein Rpl23 in Saccharomyces cerevisiae. J Biol Chem 292(2): 585-596.

174. Loar JW, Seiser RM, Sundberg AE, Sagerson HJ, Ilias N, ZobelThropp P, Craig EA, Lycan DE (2004). Genetic and biochemical interactions among Yar1, Ltv1 and Rps3 define novel links between environmental stress and ribosome biogenesis in Saccharomyces cerevisiae. Genetics 168(4): 1877-1889.

175. Graifer D, Malygin A, Zharkov DO, Karpova G (2014). Eukaryotic ribosomal protein S3: A constituent of translational machinery and an extraribosomal player in various cellular processes. Biochimie 99: 8-18.

176. Koch B, Mitterer V, Niederhauser J, Stanborough T, Murat G, Rechberger G, Bergler H, Kressler D, Pertschy B (2012). Yar1 protects the ribosomal protein Rps3 from aggregation. J Biol Chem 287(26): 21806-21815

177. Hector RD, Burlacu E, Aitken S, Bihan TL, Tuijtel M, Zaplatina A, Cook AG, Granneman S (2014). Snapshots of pre-rRNA structural flexibility reveal eukaryotic $40 \mathrm{~S}$ assembly dynamics at nucleotide resolution. Nucleic Acids Res 42(19): 12138-12154.

178. Holzer S, Ban N, Klinge S (2013). Crystal structure of the yeast ribosomal protein rpS3 in complex with its chaperone Yar1. J Mol Biol 425(22): 4154-4160.

179. Mitterer V, Murat G, Rety S, Blaud M, Delbos L, Stanborough T, Bergler H, Leulliot N, Kressler D, Pertschy B (2016). Sequential domain assembly of ribosomal protein S3 drives 40 S subunit maturation. Nat Commun 7: 10336.

180. Ghalei H, Schaub FX, Doherty JR, Noguchi Y, Roush WR, Cleveland $\mathrm{JL}$, Stroupe ME, Karbstein K (2016). Hrr25/CK1ס-directed release of Ltv1 from pre-40S ribosomes is necessary for ribosome assembly and cell growth. J Cell Biol 208(6): 745-759.

181. Larburu N, Montellese C, O'Donohue MF, Kutay U, Gleizes PE, Plisson-Chastang C (2016). Structure of a human pre-40S particle points to a role for RACK1 in the final steps of $18 \mathrm{~S}$ rRNA processing. Nucleic Acids Res 44(17): 8465-8478.

182. Merwin JR, Bogar LB, Poggi SB, Fitch RM, Johnson AW, Lycan DE (2014). Genetic analysis of the ribosome biogenesis factor Ltv1 of Saccharomyces cerevisiae. Genetics 198(3): 1071-1085.

183. Johnson MC, Ghalei H, Doxtader KA, Karbstein K, Stroupe ME (2017). Structural Heterogeneity in Pre-40S Ribosomes. Structure 25(2): 329-340.

184. Hellmich UA, Weis BL, Lioutikov A, Wurm JP, Kaiser M, Christ NA, Hantke K, Kotter P, Entian KD, Schleiff E, Wohnert J (2013). Essential ribosome assembly factor Fap7 regulates a hierarchy of RNA-protein interactions during small ribosomal subunit biogenesis. Proc Natl Acad Sci USA 110(38): 15253-15258.

185. Loc'h J, Blaud M, Rety S, Lebaron S, Deschamps P, Bareille J, Jombart J, Robert-Paganin J, Delbos L, Chardon F, Zhang E, Charenton C, Tollervey D, Leulliot N (2014). RNA mimicry by the fap7 adenylate kinase in ribosome biogenesis. PLoS Biol 12(5): e1001860.

186. Li S, Duan J, Li D, Ma S, Ye K (2011). Structure of the Shq1-Cbf5Nop10-Gar1 complex and implications for H/ACA RNP biogenesis and dyskeratosis congenita. EMBO J 30(24): 5010-5020. 
187. Walbott $H$, Machado-Pinilla $R$, Liger $D$, Blaud $M$, Réty $S$, Grozdanov PN, Godin K, van Tilbeurgh H, Varani G, Meier UT, Leulliot $N$ (2011). The H/ACA RNP assembly factor SHQ1 functions as an RNA mimic. Genes Dev 25(22): 2398-2408.

188. Leulliot N, Godin KS, Hoareau-Aveilla C, Quevillon-Cheruel S, Varani G, Henry Y, Van Tilbeurgh H (2007). The box H/ACA RNP assembly factor Naf1p contains a domain homologous to Gar1p mediating its interaction with Cbf5p. J Mol Biol 371(5): 1338-1353.

189. Vanrobays E, Gelugne JP, Caizergues-Ferrer M, Lafontaine DL (2004). Dim2p, a KH-domain protein required for small ribosomal subunit synthesis. RNA 10(4): 645-656.

190. Nissen P, Kjeldgaard M, Nyborg J (2000). Macromolecular mimicry. EMBO J 19(4): 489-495.

191. Pelava A, Schneider C, Watkins NJ (2016). The importance of ribosome production, and the 5S RNP-MDM2 pathway, in health and disease. Biochem Soc Trans 44(4): 1086-1090.

192. Lafontaine DL (2015). Noncoding RNAs in eukaryotic ribosome biogenesis and function. Nat Struct Mol Biol 22(1): 11-19.

193. Granneman S, Nandineni MR, Baserga SJ (2005). The putative NTPase Fap7 mediates cytoplasmic 20S pre-rRNA processing through a direct interaction with Rps14. Mol Cell Biol 25(23): 10352-10364.

194. Jakovljevic J, de Mayolo PA, Miles TD, Nguyen TM, Léger-Silvestre I, Gas N, Woolford JL, Jr. (2004). The carboxy-terminal extension of yeast ribosomal protein S14 is necessary for maturation of $43 \mathrm{~S}$ preribosomes. Mol Cell 14(3): 331-342.

195. Zhang J, Bai D, Ma X, Guan J, Zheng X (2014). hCINAP is a novel regulator of ribosomal protein-HDM2-p53 pathway by controlling NEDDylation of ribosomal protein S14. Oncogene 33(2): 246-254.

196. Yu YT, Meier UT (2014). RNA-guided isomerization of uridine to pseudouridine-pseudouridylation. RNA Biol 11(12): 1483-1494.

197. Massenet S, Bertrand E, Verheggen C (2016). Assembly and trafficking of box C/D and H/ACA snoRNPs. RNA Biol.
198. Li S, Duan J, Li D, Yang B, Dong M, Ye K (2011). Reconstitution and structural analysis of the yeast box H/ACA RNA-guided pseudouridine synthase. Genes Dev 25(22): 2409-2421.

199. Dez C, Noaillac-Depeyre J, Caizergues-Ferrer M, Henry Y (2002). Naf1p, an essential nucleoplasmic factor specifically required for accumulation of box H/ACA small nucleolar RNPs. Mol Cell Biol 22(20): 7053-7065.

200. Fatica A, Dlakic M, Tollervey D (2002). Naf1p is a box H/ACA snoRNP assembly factor. RNA 8(12): 1502-1514.

201. Yang PK, Rotondo G, Porras T, Legrain P, Chanfreau G (2002). The Shq1p.Naf1p complex is required for box H/ACA small nucleolar ribonucleoprotein particle biogenesis. J Biol Chem 277(47): 4523545242.

202. Grozdanov PN, Roy S, Kittur N, Meier UT (2009). SHQ1 is required prior to NAF1 for assembly of H/ACA small nucleolar and telomerase RNPs. RNA 15(6): 1188-1197.

203. Godin KS, Walbott H, Leulliot N, van Tilbeurgh H, Varani G (2009). The box H/ACA snoRNP assembly factor Shq1p is a chaperone protein homologous to Hsp90 cochaperones that binds to the Cbf5p enzyme. J Mol Biol 390(2): 231-244.

204. Darzacq X, Kittur N, Roy S, Shav-Tal Y, Singer RH, Meier UT (2006). Stepwise RNP assembly at the site of H/ACA RNA transcription in human cells. J Cell Biol 173(2): 207-218.

205. Wong CC, Traynor D, Basse N, Kay RR, Warren AJ (2011). Defective ribosome assembly in Shwachman-Diamond syndrome. Blood 118(16): 4305-4312.

206. Sulima SO, Patchett S, Advani VM, De Keersmaecker K, Johnson AW, Dinman JD (2014). Bypass of the pre-60S ribosomal quality control as a pathway to oncogenesis. Proc Natl Acad Sci USA 111(15): 5640-5645.

207. Armistead J, Triggs-Raine B (2014). Diverse diseases from a ubiquitous process: the ribosomopathy paradox. FEBS Lett 588(9): 1491-1500. 